Aus der medizinischen Klinik der Universität Leipzig (Direktor: Geh.-Rat Prof. Dr. v. Strümpell).

\title{
Klinische Beiträge zur Kenntnis der Hirnaneurysmen.
}

$$
\text { Von }
$$

\section{Privatdozent Dr. Heinrich Wichern,}

vormal. Assistent der Klinik, jetzigem leit. Arzte der inneren Abteilung des städt. Frankenhauses in Bielefeld.

Trotz der grossen Fortschritte, die in den letzten Jahrzehnten von der inneren Medizin gemacht worden sind, gibt es doch noch ganze Gruppen von Krankheiten, bei denen unsere therapeutischen Erfolge ausserordentlich gering sind. Hierher gehören insbesondere die Gefässerkrankungen, deren Behandlung bisher fast ausschliesslich in der versuchsweisen Verhütung eines Fortschreitens des schon längst in der Entwicklung begriffenen Krankheitsvorganges besteht. Die Ursache für die Machtlosigkeit unserer Therapie bei dieser Krankheitsgruppe beruht teilweise wohl auf unseren geringen Kenntnissen über die wahre Ätiologie des jeweiligen Leidens und andernteils auf dem Umstande, dass die Krankheit gewöhnlich über grosse Gefässbezirke, häufig über den ganzen Körper, ausgedehnt ist.

Aber, selbst wenn dieses letztere nicht der Fall ist, wird sich doch oft eine wirksame Behandlung deshalb als unmöglich erweisen, weil mit ihr eine Störung des Blutkreislaufs innerhalb des von dem erkrankten Gefässteile versorgten Gebietes verbunden sein würde und dadurch wichtige, ja zum Leben nötige Körperteile der Sauerstoffzufuhr beraubt würden. Diese Schwierigkeit tritt uns besonders bei den Aneurysmen entgegen, die andernfalls vielleicht häufiger durch chirurgische Eingriffe wirksam behandelt werden könnten.

Im allgemeinen dürfte das namentlich auf die Aneurysmen grosser Gefässe zutreffen; doch auch an kleineren Arterien, die lebenswichtige Organe versorgen, ist die operative Entfernung eines Aneurysma meist unausführbar. Von den mittleren oder kleineren Gefässen neigen nun aber gerade die Hirnarterien besonders häufig zur Bildung von Ausbuchtungen, und hier erscheinen die Aussichten auf eine chirurgische Behandlung recht gering.

Immerhin dürfte es wohl einzelne Fälle geben, in denen auch das Aneurysma eines Hirngefässes so günstig sitzt, dass seine Entfernung 
ohne schwere Folgen für die Blutversorgung wichtiger Hirnteile möglich oder wenigstens vorläufig denkbar ist. Praktische Versuche einer solchen Therapie sind bisher kaum ausgeführt worden, wenn man davon absieht, dass in einigen Fällen - meist mit wenig glücklichem Erfolg - eine Unterbindung der Carotis communis zwecks Heilung eines Aneurysma der Carotis interna vorgenommen wurde. Das erste und bisher das grösste Hindernis, um den Gedanken einer solchen operativen Therapie der Hirnaneurysmen wenigstens in einzelnen Fällen zu verwirklichen, bildete wohl die Schwierigkeit der Diagnose.

Aus der Literatur lässt sich eine ganze Reihe von Fällen zusammenstellen, in denen ein Hirnaneurysma klinisch überhaupt keine Erscheinungen hervorrief, sondern zufällig bei der Sektion gefunden wurde. Häufiger sind wohl die klinisch nicht sicher zu deutenden Fälle, bei denen ein bis dahin latentes Aneurysma plötzlich zu einer ausgedehnten Hirnblutung führte und infolgedessen sehr rasch den Tod bedingte.

Wenn solche Fälle vom klinischen Standpunkte aus auch vorwiegend nur statistischen Wert haben, so seien hier doch kurz zwei solcher Vorkommnisse aus der Leipziger medizinischen Klinik aufgeführt; die Sektionsprotokolle dieser und aller noch zu erwähnenden Fälle stellte mir Herr Geh.-Rat Prof. Dr. Marchand in liebenswürdigster Weise zur Verfügung, wofür ich ihm an dieser Stelle nochmals meinen besten Dank ausspreche.

Fall 1. H. G., 63 jährige Wirtschafterin, wurde bewusstlos eingeliefert; angeblich soll sie vor 3 oder 2 Jahren einen Schlaganfall gehabt haben, der in kurzer Zeit völlig ausheilte. Näheres war darüber nicht zu erfahren. Vor der Einlieferung fiel sie plötzlich um, zeigte in der Klinik tiefstes Coma, erloschene Pupillenreaktion, keine sicher nachweisbare Lähmung. Deutliche periphere Atherosklerose. Im Urin kein Eiweiss. Tod nach wenigen Stunden.

Sektion (L.-Nr. 483/02; Obduzent: Herr Dr. Risel):

Schädeldach gut ablösbar; Dura auf der rechten Seite ziemlich stark gespannt, unter ihr durchschimmerndes blaurotes Gerinnsel. Das Gehirn wird durch Horizontalschnitt zerlegt. Zwischen Dura und Oberfläche der rechten Grosshirnhälfte liegt eine besonders in den vorderen Teilen ziemlich dicke $(1 \mathrm{~cm})$ Schicht frischen, dunkelroten Coagulums. Die mediane Begrenzung der beiden Hemisphären ist etwas nach links verschoben, dadurch, dass die rechte Hemisphäre stärker ansgedehnt ist als die linke; in der Mitte misst die Breite der rechten Hemisphäre etwa 7, die der linken $6 \mathrm{~cm}$. Die grossen Ganglien sind rechts ziemlich stark geschwollen und auf dem Durchschnitt rechts etwa $31 / 2$, links nur $21 / 2 \mathrm{~cm}$ breit. Der rechte Seitenventrikel ist vollständig verstrichen, ebenso das Vorderhorn des linken, während das Hinterhorn ziemlich weit und mit klarer Flüssig- 
keit gefüllt ist. Bei der Herausnahme des unteren Abschnittes des Gehirns findet sich an der Basis der rechten Hemisphäre ebenfalls eine dicke Schicht dunkelroten Coagulums, das mit dem vorerwähnten zusammenhängt und sich weiter nach der Konvexität hinauf erstreckt. Sehr reichlich erscheinen diese Gerinnsel an der Spitze des rechten Schläfenlappens. Etwas nach hinten und medianwärts hiervon ist die Hirnsubstauz in einem etwa markstückgrossen Bereiche zerstört durch blutige Gerinnsel, die sich tief in sie hineingewühlt haben. Das ist in der Weise geschehen, dass sie von der medialen Fläche her schräg nach hinten und aussen die Gehirnsubstanz durchbrochen haben, so dass nur eine schmale Brücke erhaltener Gehirnsubstanz über die zerstörte Masse hinwegreicht. Beim Präparieren der Gefässe findet sich etwas seitlich von der rechten A. communicans post. ein kleiner aneurysmatischer Sack (10-11 mm lang), der sich unmittelbar hinter der Abgangsstelle der rechten $A$. fossa Sylvii von der A. carotis nach hinten erstreckt, ohne selbst einem kleinen Aste der Art. fossa Sylvii anzugehören. Dieser in die Blutgerinsel eingebettete Sack ist mit der Dura am hinteren rechten Processus clinoides fest verwachsen, so dass bei der Herausnahme der untere Teil seiner Wand durchschnitten wird. In seinem hinteren Abschnitte findet sich das Lumen durch gelbliche Thrombusmassen ausgefüllt; die Perforationsstelle selbst ist nicht mehr deutlich nachzuweisen, scheint aber an diesem hinteren Ende zu liegen. Die Arterie zeigt im übrigen sehr wenige gelbliche Verdickungen. Der N. oculomotorius zieht über den Sack hin und ist ebenfalls mit seiner Wand ziemlich fest verbunden; zugleich erscheint er stark verbreitert und abgeplattet.

Sektionsdiagnose: Atherosclerosis arteriar. cerebri. Aneurysma sacciforme art. fossae sylvii dextrae. Haematoma intermeningeale dextr. et haemorrhagia cerebri (lobi temporal. dextr.) ex ruptura aneurysmatis. Emphysema et tuberculosis obsoleta pulmon. Bronchitis chron. Hypertrophia ventriculi dextri cordis. Phthisis renalis sin. Tuberculosis et ulcera pelvis renis sin., ureteris sin. et vesicae urinariae.

Fall 2. K. N., 44jähr. Restaurateur, soll reichlich Alkoholmissbrauch getrieben haben, hat schon länger Allgemeinbeschwerden, ist plötzlich umgefallen, bewusstlos geworden und wird sterbend ins Krankenhaus eingeliefert. Keine Lähmungssymptome. Etwas Zucker im Urin.

Sektion (L.-Nr. 548/06; Obduzent: Herr Dr. Versé):

Schädeldach länglich, stark gewölbt, ziemlich breit. Dura fest adhärent. An der rechten Seite finden sich hämorrhagische Infiltrationen der weichen Häute, die anscheinend von der Fossa Sylvii ausgehen und sich nach allen Richtungen verbreiten. Ebenso sind die Häute au der Basis um den Hirnstamm stark mit Blut durchsetzt, so dass sie die Konfiguration verdecken, ebenso zwischen den Stirnlappen und um das Chiasma opticum herum. Beim Durchschneiden des Gehirns in horizontaler Richtung kommt ein grosser, mit dunkelrotem Coagulum gefüllter Herd zum Vorschein, der in der rechten Fossa Sylvii, sowie zwischen Stirn- und Schläfenlappen gelegen ist und sich nach hinten in die Hirnsubstanz selbst bis ins Unterhorn hinein erstreckt. In den Ventrikeln findet sich blutig gefärbte Flüssigkeit, die bereits aus dem Infundibulum bei der Herausnahme des Gehirns hervorquoll. Beim Herausschälen der Blutmassen aus den weichen Häuten 
der Basis wird in der Gegend der A. cerebri media dextra ein nach unten sich vorwölbender, knopfartiger Vorsprung sichtbar, an dem festere Coagula haften. Er sitzt an der Hauptteilungsstelle der Arterie und erweist sich als eine erbsengrosse Ausbuchtung der Arterienwand, in die man mit der Sonde vom Hauptstamm aus leicht hineingelangt. Ein kollabierter Gefässast geht gerade von dieser Ausbuchtung ab. Sie selbst wölbt sich gegen die Schädelbasis stark vor und zeigt hier eine glatte Wandung. Nach dem Gehirn zu, also an der Basis der Ausbuchtung gelangt man mit einer Sonde durch eine Perforation hindurch.

Sektionsdiagnose: Ruptura aneurysmatis sacciformis art. cerebri mediae dextrae. Infiltratio haemorrhagica leptomeningum praecipue fossae Sylvii dextrae et baseos cerebri. Haemorrhagia in lobum temporale dextrum. Perforatio cornu infer. ventric. lateral. dextri. Contenta sanguinolenta ventriculi cerebri. Dilatatio magna et hypertrophia mediocris cordis. Hepatitis interstitialis chron. (Cirrhosis hepatis incip.) Atrophia granularis incip. renum. Degeneratio adiposa tunicae intimae et atherosclerosis levis aortae. Pleuritis chron. adhaesiva bilateralis. Hernia inguinalis ext. sin.

In beiden Fällen trat ohne besondere Vorboten plötzlich ein apoplektiformer Insult auf, führte sofort zu schwerer Bewusstlosigkeit und binnen wenigen Stunden zum Tode. Es handelte sich dabei im ersten Falle um eine ältere Frau mit nachweisbarer Atherosklerose und im zweiten um einen Mann in mittlerem Alter, der aber stark dem Alkohol zugesprochen haben sollte und deshalb zu apoplektischen Anfällen sicherlich disponiert sein musste. Unter diesen Umständen war die Wahrscheinlichkeit einer einfachen Gefässzerreissung im Gehirn mit profuser Blutung zweifellos grösser, als die eines Aneurysma, und es wird in solchen Fällen niemals möglich sein, klinisch die Diagnose einer Hirngefässerweiterung zu stellen. Über den früheren Schlaganfall der Frau (Fall 1) fehlten alle näheren Angaben, so dass daraus ebenfalls keinerlei klinische Schlüsse, wie sie weiterhin noch für die Hirnaneurysmen zu erörtern sein werden, zu ziehen waren.

Als Ursache der Gefässerweiterung haben wir wohl in beiden Fällen atherosklerotische Gefässveränderungen anzusprechen, wie wir sie ja im mittleren und höheren Alter so häufig an den Hirnarterien finden, auch ohne dass sich bestimmte, dazu führende Schädigungen aus der Anamnese ergeben. Wir haben leider kaum jemals genügende Anhaltspunkte dafür, wann im einzelnen Falle die Verhärtung der Arterien begonnen hat, und es fehlt uns auch wohl immer eine Schätzung darüber, innerhalb welcher Zeit sich ein Aneurysma von bestimmter Grösse auf atherosklerotischer Grundlage entwickelt hat oder entwickeln kann. Selbst bei verhältnismässig jugendlichen Personen kommt ja schon eine ausgedehntere Verhärtung der cere- 
bralen Gefässe vor und kann vermutlich sehr lange bestehen bleiben, ohne dass das Leben gefährdet wird oder sichere klinische Erscheinungen dieses Leidens hervortreten.

Die Kenntnis dieser Tatsachen erschwert es uns also sehr, einen Maßstab für das Alter des einzelnen Aneurysma zu gewinnen, selbst wenn wir mit Sicherheit behaupten können, dass dieses sich erst infolge atherosklerotischer Veränderungen gebildet hat. Wie vorsichtig man aber auch mit dieser Annahme sein muss, mag uns folgendes Beispiel, dessen klinischer Verlauf wiederum nur geringes Interesse hat, lehren:

Fall 3. E. 0., 37jähriger Arbeiter, ist plötzlich bewusstlos umgefallen und wird unter den Erscheinungen einer rechtseitigen Hemiplegie eingeliefert. Der Zustand verschlechtert sich sehr schnell, so dass Pat. schon nach kurzer Zeit stirbt.

Sektion: (L.-Nr. 1448/08; Obduzent: Herr Dr. Müller):

Schädeldach oval, mitteldick, glatt. Nähte undeatlich, Dura ablösbar. In den Sinus wenig dickflüssiges Blut. Dura mittelmässig gespannt, und zwar beiderseits gleichmässig, Innenfläche glatt. Bei der Herausnahme des Gehirns zeigen sich an der Basis grosse Mengen ausgetretenen Blutes zwischen den weichen Hänten, das namentlich in der Gegend des Chiasma und des Pons sich ausbreitet und hanptsächlich die Basis des linken Schläfenlappens einnimmt. Die Hirnarterien zeigen auf dem Durchschnitt eine rigide Wand und klaffen; in dem Winkel zwischen der linken Crotis interna und der A. communicans post. sin. findet sich ein medial sich vorwölbender, $2,2 \mathrm{~cm}$ langer und $1,7 \mathrm{~cm}$ breiter Sack, der mit der linken Carotis in enger Verbindung steht. Die Wand des Sackes ist unten sehr dünn und zeigt keine sklerotischen Veränderungen. An seiner linken Seite finden sich festere, dunkelrote Coagula, die mit dem Sacke etwas zusammenhängen. Hier findet sich auch im hinteren Teile ein für eine dünne Sonde durchgängiges Loch in der Wand. Durch diesen Sack wird der Stiel der Hypophyse nach rechts hinübergedrängt. Der linke Nervus oculomotorius zieht über den Sack hinweg. Die Blutungen zwischen den weichen Häuten erstrecken sich weit nach der Konvexität hinauf und namentlich in die linke Fossa Sylvii hinein, wo sie eine etwa $3 / 4 \mathrm{~cm}$ dicke Schicht bilden. Ein Horizontalschnitt durch das gehärtete Gehirn ergibt keine Veränderungen an den Ganglien oder in der übrigen Hirnsubstanz.

Seltionsdiagnose: Aneurysma art. carotid. int. sin. magnum perforat. Infiltratio haemorrh. vasta leptomeningea. Dilatatio vesicae urinariae. Pneumonia lobularis incipiens et oedema haemorrh. pulm. Degeneratio adiposa tunicae intimae aortae et arteriar. magnarum.

Die Sektion wies in diesem Falle eine für das Alter des Kranken (37 Jahre) recht erhebliche Atherosklerose der Hirngefässe nach, wie sie klinisch wohl kaum zu vermuten war. Es liegt also der Gedanke nahe, dass auf dem Boden dieses Leidens auch das Aneurysma der linken Carotis interna entstanden war. Auffällig ist demgegenüber aber, dass gerade die Wandung der erweiterten Stelle Verkal- 
kungen oder Einlagerungen vermissen liess; auch legt die schon recht beträchtliche Grösse des Aneurysma $(2,2 \times 1,7 \mathrm{~cm})$ die Frage nahe, ob nicht der Ursprung dieser Gefässgeschwulst schon in eine sehr viel frühere Zeit zu verlegen ist, als der erste Beginn der übrigen Gefässerkrankung.

Wir würden es dann also wohl mit einem sog. kongenitalen Aneurysma zu tun haben, das sich eben auf einer angeborenen Anlage allmählich zu immer grösserem Umfange entwickelt hat. Fälle, bei denen man zwischen der Entscheidung einer kongenitalen oder atherosklerotischen Entstehungsweise zu wählen hat, sind überhaupt nicht so ganz selten, und im allgemeinen scheint die Neigung, bei Vorhandensein einer Aderverkalkung diese ohne weiteres auch als Ursache für ein bestehendes Hirnaneurysma hinzustellen, grösser zu sein, als die Befunde und Tatsachen zu solcher Annahme berechtigen. Gerade bei aussergewöhnlich grossen Aneurysmen, die doch wohl eine lange Entwicklungszeit hinter sich haben, ist es sehr zu überlegen, ob die nebenher gefundene Atherosklerose nicht ein sekundärer Vorgang war, der natürlich auch die Wandung des Aneurysma ergreifen und damit eine atherosklerotisch entstandene Ausbuchtung vortäuschen kann. Soucques fand bei einem 65jährigen Manne, der durch Selbstmord endete, neben reichlicher Atheromatose der Hirngefässe ein über hühnereigrosses Aneurysma der rechten A. cerebri media, dessen Entstehung nach der Krankengeschichte wohl zweifellos schon 55 Jahre zurücklag! Wir dürfen ja nicht vergessen, dass uns daneben Beobachtungen zur Verfügung stehen, wo bei älteren Leuten mit ganz zartwandigen Hirngefässen kleine Hirnaneurysmen vorhanden sind oder zur Perforation gelangen, für die kaum eine andere Ätiologie, als die Annahme kongenitaler Gefässveränderungen in Frage kommt. An dieser Stelle dürfte daher auch folgendes Beispiel von Interesse sein, dass geradezu zur Erläuterung dieser letzten Ausführungen dienen kann:

Fall 4. B. W., 56 jährige Tischlersehefrau, war bis dahin im allgemeinen ganz gesund, fiel früh plötzlich in der Wohnung um, klagte über starke Kopfschmerzen und erbrach mehrere Male. Bei der Aufnahme in die Klinik war sie schwer besinnlich, wies keinerlei Lähmungssymptome auf, vermochte sich aber nicht aufzurichten. Nach etwa 16 Stunden erfolgte unter zunehmender Bewusstlosigkeit ohne Auftreten besonders bemerkenswerter Erscheinungen der Tod.

Sektion: (L.-Nr. 992/09; Obduzent Herr Dr. Knierim):

Schädeldach symmetrisch, mässig dick, innen glatt. Dura mater ziemlich prall, über beiden Hemisphären gleichmässig gespannt, Sinus mit Blut gefüllt. Pia der Konvexität durchsichtig, Venen gut gefüllt. An der Basis finden sich, besonders in den Maschen der Pia, dunkelrote Cruormassen, 
die Alächenhaft die Gegend der Bräcke, der Medulla oblongata und der medialen unteren Teile der Kleinhirnhemisphären, sowie das Chiasma opticum bedecken. Die Gefässe der Basis haben eine sehr dünne Wandung; in der Wand der linken A. cerebelli post. inf. findet sich eine winklige Öf fnu ng, an der sich ein Teil der Wand lappenartig emporhebt. Hier liegen ebenfalls reichliche Cruormassen, die sich durch das Foramen Magendii in den vierten Ventrikel fortsetzen, so dass dieser ganz von ihnen erfüllt ist. Auch im Hinterhorn der Seitenventrikel findet sich etwas Cruor. Das Gehirnparenchym selbst zeigt keine pathologischen Veränderungen.

Die Dura mater des Rückenmarks ist prall gespannt, schimmert blaurot durch. Unter der Arachnoidea finden sich bis etwa zum Ende des Spinalkanals noch reichliche Cruormassen und etwas flüssiges Blut.

Sektionsdiagnose: Dilaceratio aneurysmatis parviart. cerebelli post. inf. sin. Infiltratio haemorrhagica leptomeningam baseos cerebri et medullae spinalis. Contenta sanguinolenta ventricali quarti cerebri et cornuum post. ventric. lat. Haemorrhagiae diffusae subpleurales. Peritonitis et perisplenitis chron. fibrosa circumscripta. Cystis renis dextri.

Trotz etwas vorgeschrittenen Alters fand sich also bei dieser Frau keine Spur cerebraler Atherosklerose und dennoch hatte sich an der Art. cerebelli post. inf. sin. ein allerdings nur sehr kleines Aneurysma gebildet, das schliesslich geborsten war. Es fehlt uns in diesem Falle jeder Anhaltspunkt dafür, wie lange die Ausbuchtung schon bestanden haben mag; ich möchte es für wahrscheinlich halten, dass sie schon in der Jugend vorhanden war und erst durch langsame Zunahme der Verdünnung ihrer Wand an einer Stelle schliesslich zum Platzen kam, wofür ein Beweis jedoch nicht zu liefern ist.

Vor allem hat ja Eppinger in seiner ausführlichen Arbeit über Aneurysmen auf die kongenitale Anlage zur Bildung solcher Geschwïlste an den Hirngefässen aufmerksam gemacht. Bei einer genaueren Durchsicht der Literatur gelang es mir festzustellen, dass etwa 24,5 Prozent aller Hirnaneurysmen, wenn nicht mehr, auf diese Weise zu erklären sein dürften. ${ }^{1)}$ Während manche der so entstandenen Gefässgeschwülste offenbar eine ganz enorme Grösse (Fall Oppe, Roe, Kingston u. a.) erreichen können, ohne zu platzen, gelangen andere schon frühzeitig zur Berstung und rufen dann erst mit dieser und daher oft nur unmittelbar vor dem Tode klinische Erscheinungen hervor. Nachstehende Beobachtungen, von denen die zweite recht bemerkenswert erscheint, mögen neben anderen, aus der Literatur bekannten, als Beispiele dienen.

Fall 5. M. D., 21jährige Schneiderin, ist bewusstlos geworden und kommt in diesem Zustande in die Klinik. Mehrfach werden bei ihr klonische und tonische Krämpfe der Muskeln aller Extremitäten beobachtet.

1) s. Wichern, Münch. med. Wochenschr. 1911. Nr. 51. 
Lähmungserscheinungen fehlen. Im Urin kein Eiweiss und Zucker. Kurz nach der Aufnahme Tod.

Sektion: (L.-Nr. 943/07; Obduzent: Herr Privatdozent Dr. Risel):

Schädeldach zeigt deutliche Nähte, ist leicht abzulösen, dünn. Dura mater beiderseits sehr stark gespannt, liegt der Hirnoberfläche ganz glatt an. Im Sinus longitudinalis nur Leichengerinnsel. Innenfläche der Dura glatt, glänzend.

Das Gehirn ist sehr ausgedehnt; die Windungen an der Oberfläche sind deutlich und beiderseits gleichmässig verbreitert, abgeflacht, die Zwischenfurchen sehr schmal, fast verstrichen. Die Arterien an der Hirnbasis sind überall sehr zart und dünn; nirgends sind Knötchen oder Einlagerungen erkennbar. In den weichen Häuten an der Basis findet sich seröses Exsudat. Das Infundibulum ist nach unten vorgewölbt, die Hypophyse ganz in die Sella turcica hineingedrückt, während die Kleinhirntonsillen nach abwärts in den Spinalkanal gedrängt sind. Von den Zisternen des Kleinhirns aus verbreitet sich an der Unterfläche der Brücke und des Kleinhirns blutige Flüssigkeit in den weichen Häuten und ebenso rom Foramen Magendii aus.

Das Gehirn wird durch einen Horizontalschnitt durchschnitten; dabei zeigt sich in den hinteren Teilen des Stirnlappens, noch ein wenig auf den Streifenhügel und den vorderen Teil der inneren Kapsel übergreifend, ein reichlich walnussgrosser, frischer, mit dunkelroten Blutgerinnseln ausgefüllter Herd, der breit in den rechten Seitenventrikel durchgebrochen ist. Dieser ist daher, wie auch der linke Seitenventrikel und der dritte, sowie der vierte Ventrikel, prall mit frischem, dunkelroten Blutcoagulum gefüllt, das zum Teil auch noch die angrenzenden, sehr weichen Hirnhäute etwas zerstört hat. In dem frischen Coagulum des Stirnlappens findet sich ein reichlich erbsengrosses, sackartiges, kugeliges und bräunlichrotes Gebilde, dessen dünne Wand an einer Seite breit eingerissen ist und das mit einem kleinen Gefäss für eine kurze Strecke zusammenhängt. Der Zusammenhang mit einem Aste der Art. cerebri ant. ist aber jetzt nicht mehr nachweisbar. Die übrige Hirnsubstanz ist weich, zeigt keine Besonderheiten.

Sektionsdiagnose: Ruptura aneurysmatis rami art. cerebri ant. (?) dextr. Haemorrhagia magna recens lobi frontalis hemisphaeri dextri cerebri in ventriculum lateralem perforans. Haemocephalus internus. Tuberculosis caseosa lymphoglandular. perigastr., Tuberculosis recens lymphogland. cervicalium. Cystis ovarii sin. Struma colloides. Degeneratio adiposa levis tunicae int. aortae. Hyperaemia pulmon., lienis, hepatis, renum. Aspiratio contenti ventriculi. Status uteri menstrualis. Corpus rubrum ovarii dextri.

Fall 6. P. P., 32jähriger Mann, wurde im 10. Lebensjahre plötzlich von einer rechtsseitigen Lähmung der Extremitäten befallen, die mit Sprachstörung einherging. Allmählich trat eine Besserung ein, und es blieb nur eine leichte Parese des Armes und Beines zurück. Mit 31 Jahren brach Pat. plötzlich nach einer Aufregung bewusstlos zusammen, erbrach mehrmals und wurde in die Klinik geschafft. Hier wurde Herabhängen des rechten Mundwinkels und Lähmung des rechten Armes and Beines, die in Kon- 
trakturstellung sich befanden, festgestellt. Nach Wiedererlangung des Bewusstseins konnte Pat. nur wenig und unverständlich sprechen; auch vorgesprochene Worte konnten nur mangelhaft wiederholt werden. Die Intelligenz erschien ziemlich stark herabgesetzt. An den gelähmten Extremitäten bildete sich eine deutliche Atrophie aus. Für kurze Zeit trat während der klinischen Beobachtung, die fast ein halbes Jahr dauerte, eine linksseitige Oculomotoriusparese auf; Pat. wurde schliesslich mit deutlicher Hemiparese, an der auch der rechte $\mathrm{N}$. facialis noch leicht beteiligt war, entlassen. Sprachvermögen und -verständnis blieben sehr unvollkommen. Gerade ein Jahr später, also mit 32 Jahren, wurde Pat. unter den Zeichen eines neuen schweren Insultes wieder in die Klinik eingeliefert, erlangte das Bewusstsein nicht wieder, sondern starb schon am zweiten Tage.

Sektion: (L.-Nr. 257/06; Obduzent: Herr Dr. Löhlein):

Schädeldach längsoval, ziemlich symmetrisch, mässig schwer, zeigt verhältnismässig reichliche spongiöse Substanz und tiefe Pachionische Gruben. Die Dura ist mässig gespannt und scheint über der linken Hemisphäre in grösserer Ausdehnung bläulich durch; ihre Innenfläche ist glatt. Die Pia mater ist an der Konvexität des Gehirns fast in ganzer Ausdehnung blutigserös infiltriert, und zwar am meisten in der Nachbarschaft der grossen Gefässe, weniger in der Umgebung des Sinus longitudinalis. Der linke Schläfenlappen ist vollständig von einer blauroten Masse eingehüllt. An der Basis des Gehirns sammelt sich bei der Herausnahme reichlich blutigseröse Flüssigkeit an, und die Pia mater des Kleinhirns ist ebenfalls blutig infiltriert, während sich aus dem Foramen Magendii ausser flüssigem Blute auch lockere Gerinnsel entleeren. Die Arterien der Hirnbasis sind zartwandig und weich. Das Gehirn selbst zeigt nach der Herausnahme eine etwas weiche Konsistenz; namentlich die linke Gehirnhälfte fühlt sich schwappend an und sinkt, wenn das Gehirn auf der Basis aufliegt, stärker zusammen, als die rechte. Die Windungen des Grosshirns sind breit, die Furchen fast verstrichen. Auf dem Durchschnitt der Medulla oblongata zeigt sich, der Lage der linken Pyramidenbahn entsprechend, ein grau durchscheinender, scharf begrenzter, auf der Schnittfläche keilförmiger Streifen, der sich nach aufwärts auch in dem etwas eingesunkenen Hirnschenkel der gleichen Seite verfolgen lässt.

Das Hirn wird etwas vor den Hirnschenkeln durch einen Frontalschnitt zerlegt, wobei sich reichlich blutig gefärbte Flüssigkeit und lockeres Coagulum entleert. Beide Seitenventrikel, besonders der linke, zeigen sich stark dilatiert und prall mit geronnenem und flüssigem Blut gefüllt. Auf der vorderen Schnittfäche des Gehirns findet sich links ein von oben nach unten $6 \mathrm{~cm}$, seitwärts nur $3 \mathrm{~cm}$ messender Herd, der an seinen äusseren Rändern eine unregelmässig zackige Gestalt und teils bräunlichen, teils gelblichen Saum hat. Nach dem Ventrikel zu liegt ein die Hälfte der Schnittfläche einnehmendes lockeres und maschiges Gewebe von rötlicher bis bläulicher Farbe, in dem deutlich weite Gefässöffnungen sichtbar sind. Unmittelbar nach vorne von der Schnittfläche erscheint ein etwa kirschgrosser, blutig gefärbter, rundlicher und sackartiger Körper, der in das Lumen des Seitenventrikels frei hineinragt. Fr wird zum grössten Teile durch einen vom Ependym des Seitenventrikels überzogenen Sack gebildet, der mit dem grösseren Herde anscheinend nicht in Verbindung steht. Dieser Sack ist von lockeren Blutgerinnseln teilweise ein- 
gehüllt und durch eine kleine öffnung mit einer feinen Sonde zugänglich. Auch im Duralsack findet sich reichlich stark blutige Flüssigkeit.

Sektionsdiagnose: Aneurysma racemosum rami arteriae fossae Sylvii sin. (?) Haemorrhagia obsoleta et recens hemisphaerii sin. cerebri. Degeneratio grisea secundaria funiculorum descendentium lateris sin. Atrophia musculorum lateris dextri corporis.

Bei dem jugendlichen Alter beider Kranken und bei der zarten Beschaffenheit der Hirngefässe kann in beiden Fällen an der Annahme eines auf kongenitaler Grundlage entstandenen Aneurysma kaum ein Zweifel sein, zumal für eine Embolie keine genügenden Anhaltpnnkte vorhanden waren. Im zweiten Falle (Fall 6) könnte man vielleicht geradezu von einer Gefässmissbildung innerhalb des Gehirns sprechen, da es sich um ein vielgestaltiges Aneurysma mit mehreren Gefässlumina handelte. Bemerkenswert ist übrigens bei beiden Beobachtungen, dass die Gefässerweiterung innerhalb der Hirnsnbstanz selbst lag; im allgemeinen pflegen ja die Aneurysmen weit häufiger an der äusseren Oberfläche, besonders an der Basis des Gehirns, ihren Sitz zu haben, woranf später noch einmal zurückzukommen sein wird.

Während die atherosklerotischen Veränderungen durch das Brüchigwerden des Gefässrohrs das Auftreten einer Blutung gerade an der Stelle einer Erweiterung leicht erklärlich machen, müssen wir bei den auf kongenitaler Anlage entstandenen Aneurysmen eine allmählich zunehmende Verdünnung ihrer Wand, besonders an einer Stelle, annehmen und können dann ein plötzliches Einreissen, auch schon bei einem ganz geringfügigen Anlasse, verstehen. In solchen Fällen wird dann eine sehr ausgedehnte Zerreissung an der Rupturstelle eintreten, und es lässt sich nachträglich bei der Sektion kaum etwas über die Beschaffenheit der Gefässwand an der Rissstelle aussagen. Infolgedessen gestaltet sich dann zuweilen die Begutachtung eines solchen Falles ziemlich schwierig, wenn es festzustellen gilt, ob ein Trauma erst zur Perforation des Aneurysma führte, wofür u. a. v. Hoffmann zwei bemerkenswerte Beispiele anführt. Diesen reiht sich folgender Fall der Leipziger Klinik an:

Fall 7. R. G., 38jähriger Fuhrwerksbesitzer, war bisher völlig gesund und fiel plötzlich während des Fahrens vom Wagen herab. Seitdem war er völlig bewusstlos, zeigte tiefe stertoröse Atmung, aber keine Lähmung. Tod nach wenigen Stunden.

Sektion: (L.-Nr. 1117/03; Obduzent: Herr Dr. Risel):

Schädeldach verhältnismässig breit, fest anhaftend. Unter der Dura schimmern beiderseits, besonders links, blaurote, blutige Massen hindurch, die Dura selbst ist stark gespannt, namentlich links. Das Gehirn wird durch einen Horizontalschnitt zerlegt, wobei sich reichlich blutige Flüssig- . 
keit entleert. Der dritte Ventrikel ist ziemlich breit und mit frischem, dunkelrotem Coagulum gefullt, die Seitenventrikel sind weit, aber leer. Im übrigen zeigt der Durchschnitt durch das Gehirn keine Veränderungen.

In den Maschen der weichen Häute am äusseren Umfange des Gehirns findet sich uberall frisches, dunkelrotes Coagulum und flüssiges Blut. Bei der Herausnahme der Hirnbasis aus dem Schädel sind die weichen Häute an der Unterfläche beider Stirn- und Schläfenlappen, namentlich in den Furchen, ebenfalls mit frischem Blutextravat erfüllt; die Windungen selbst sind überall noch deutlich ausgeprägt. Die Gegend des Chiasma opticum und des Infundibulum ist von einem dicken Mantel von Blut umgeben, der nach der Unterfläche des Pons zu immer dicker wird; er hüllt diesen, sowie die austretenden Nerven völlig ein und erreicht in der Gegend der Pyramidenkreuzung eine Dicke von nahezu $1 \mathrm{~cm}$. In der Falte zwischen den beiden Kleinhirnhälften reicht der an Dicke rasch abnehmende Bluterguss bis zum hinteren Umfange des Wurmes; das Foramen Magendii ist noch vollständig von dicken Blutmassen überdeckt. Dagegen sind die Oberfläche der Kleinhirnhemisphären und die Kleinhirnschenkel fast vollkommen frei von Blut. In der Fossa Sylvii erstreckt sich der Bluterguss beiderseits weit nach aufwärts, und auch an der Konvexität beider Hemisphären sind die Furchen breit mit dunkelrotem, geronnenem Blut angefüllt. Die weichen Häute über den Windungen sind etwas rötlich verfärbt. Die Innenfläche der Dura ist völlig glatt, glänzend und frei von Pseudomembranen oder Blutmengen.

Gerade auf der Schnittfiäche durch die Merdulla oblongata, welche diese etwa in der Gegend der Pyramidenkreuzung trifft, liegt an der rechten Art. vertebralis ein etwa $3-4 \mathrm{~mm}$ im Durchmesser betragendes Säckchen, das mit dem sehr dünnen Gefässe in Verbindung steht und gerade in der Mitte durchschnitten ist. Die Arterien an der Basis sind überall zart und dünnwandig und zeigen nirgends Verdickungen oder Einlagerungen. Die rechte Art. vertebralis ist auffallend schmal. Der Subarachnoidalraum im ganzen Verlaufe des Rückenmarks ist mit dunklem, geronnenen oder flüssigen Blute angefüllt.

Sektionsdiagnose: Ruptura aneurysmatis sacciformis arter. vertebralis dextrae. Haematoma intermeningeale cerebri, praecipue baseos et medullae spinalis. Emphysema et oedema pulmonum.

Es handelte sich hier um die Entscheidung, ob der Fall vom Wagen durch das Platzen des Aneurysma bedingt war oder ob dieses erst infolge des Falles zum Bersten kam und so den Tod herbeiführte. Ein bestimmtes Urteil kann in einem solchen Falle wohl nur dadurch ermöglicht werden, dass Augenzeugen über den Hergang des Unfalles berichten; denn es wird wesentlich darauf ankommen, ob für den Fall vom Wagen eine andere Ursache nachzuweisen war. Fehlte diese, so ist die Wabrscheinlichkeit grösser, dass das Aneurysma schon zum Bersten reif war und durch seine Ruptur dann erst das Herabfallen des Kranken verursachte; bei dieser letzteren Annahme ist gutachtlich das Bestehen einer Unfallsfolge natürlich abzulehnen. Häufiger und wichtiger wird für den ärztlichen Begutachter aber 
die Frage sein, ob ein bei der Sektion gefundenes Hirnaneurysma mit einem meist schon mehrere Jahre zurückliegenden Schädeltrauma in ursächliche Beziehung zu bringen ist. Es lässt sich ja nicht bezweifeln, dass Gefässerweiterungen, namentlich an der Unterfläche des Gehirns, durch Aufprallen der Gehirnmassen auf die Sehädelbasis entstehen können; es kommt in solchen Fällen wohl meist zur Zerreissung einzelner Teile der Arterienwand, besonders der Elastica, und nun kann sich durch den ällmählichen. Druck von innen her eine Ausbuchtung an der geschädigten Stelle entwickeln. Besonders lehrreich ist in. dieser Beziehung ein von Saathoff beschriebener Fall, bei dem der Tod bald nach dem Unfall erfolgte und die Sektion einen breiten Einriss der Media an der Art. basilaris erkennen liess. Auch eine von Gull erwähnte Beobachtung (1. c. Fall 1) dürfte hierher gehören.

Trotz dieser sehr beweiskräftigen Beispiele glaube ich doch, dass man 'mit der Annahme eines Zusammenhangs zwischen Trauma und Hirnaneurysma sehr zurückhaltend sein muss. Leichtere Schädeltraumen sind sehr häufig und können deshalb kaum für unsere Frage in Betracht kommen; bei schwereren Unfällen wird aber nur dann das Aneurysma als Folgeerscheinung anzusehen sein, wenn der ursprüngliche Riss innerhalb der Arterienwand noch nachweisbar ist oder sonstige Zeichen klinischer und pathologisch-anatomischer Art dartun, dass auch die Meningen oder einzelnen Gehirnteile bei dem Unfall stärker komprimiert worden sind. Es wird zugleich bei der Sektion darauf zu achten sein, ob nicht an einigen der übrigen Hirnarterien ebenfalls kleine Ausbuchtungen vorhanden sind, was dann entschieden mehr für eine Entstehung auf kongenitaler Grundlage als durch Trauma sprechen würde.

Eine der häufigsten und wichtigsten Ätiologien für Hirnaneurysmen gerade bei jugendlichen Personen ist die Embolie mit infektiösem Material. Im Verlaufe einer ulzerierenden Endokarditis kann, wie Ponfick und Simmonds gezeigt haben, ein mit Mikroorganismen beladener Pfropf in eine der Hirnarterien gelangen und sich dort festsetzen, ohne dass dabei das Lumen völlig verstopft wird und deshalb sichtbare klinische Erscheinungen auftreten müssen. Die Keime wandern in die Arterienwand ein, zerstören sie, und infolgedessen buchtet sie sich immer stärker aus, bis schliesslich die Ruptur erfolgt. Wir hatten Gelegenheit, mehrere solche Beispiele zu beobachten und die Krankengeschichțen mögen hier berichtet sein:

Fall 8. M. Str., 40jährige Witwe, soll schon Jahre lang leidend sein und ist seit November 1907 bettlägerig. Am Abend vor der Aufnahme (10. Febr. 1908) wurde sie bewusstlos aufgefunden. Bei der grazil ge- 
bauten, etwas cyanotischen Kranken konnte eine schlaffe Lähmung der rechten Seite und ein Herabhängen des rechten Mundwinkels festgestellt werden. Der Herzspitzenstoss befand sich ausserhalb der Mammillarlinie im 5. Intercostalraum, war verbreitert und hebend; an der Spitze lautes systolisches Geräusch, 2. Pulmonalton klappend, rechte Herzgrenze 1 Querfinger ausserhalb des rechten Sternalrandes.

Während der folgenden Tage keine wesentliche Änderung ausser eines plötzlichen Temperaturanstiegs auf $40,3^{\circ}$ am 12. Februar; dauernd tiefes Coma. Am 14. Februar 1908 Exitus.

Sektion: (L.-Nr. 232/08; Obduzent: Herr Dr. Löhlein):

Der Schädel ist ziemlich flach, das Schädeldach schwer. Keine Verwachsungen mit der Dura; diese selbst ist ziemlich stark gespannt ohne merklichen Unterschied zwischen beiden Hemisphären. Innenfläche der Dura glatt; die Pia zeigt eine ziemlich starke Füllung der Gefässe und im Bereiche des linken Stirn- und Schläfenlappens breite hämorrhagisch infiltrierte Streifen, die den Gehirnfurchen entsprechen. Die Windungen des Gehirns sind überall breit, die Furchen sehr schmal, fast verstrichen. Die Gefässe an der Basis sind durchaus zart. Die weichen Häute der Basis des linken Stirn- und Schläfenlappens sind diffus leicht hämorrhagisch infiltriert und zeigen gleichmässig rötliche Farbe. Die Fossa Sylvii und ihr benachbarte Stellen enthalten reichliche, schwarzrote, lockere und geronnene Blutmassen. Der Stamm der Art. fossa Sylvii sin. ist ganz frei; etwa $6 \mathrm{~cm}$ von der Abgangsstelle entfernt findet sich an einem der stärksten Äste der Arterie, der an dem vorderen Pole des Schläfenlappens verläuft, ein etwa erbsengrosses, graurötliches und sackförmiges Gebilde, an dessen glatter Wand der Arterienast fest adhärent ist. Die ganze vordere Hälfte des Schläfenlappens fühlt sich sehr weich an. Beim Präparieren reisst die Oberfläche ein, und es entleeren sich weissliche Fetzen erweichter Gehirnsubstanz. Aus dem linken Seitenventrikel quellen lockere Blutcoagula hervor; auf einem Horizontalschnitt durch das Gehirn zeigt sich im Schläfenlappen ein kleinapfelgrosser, hämorrhagischer Herd, der bis an das Unterhorn des Seitenventrikels heranreicht.

Sektionsdiagnose: Endocarditis chron. ulcerosa valvulae mitral. et aortae. Insufficientia valvul. mitral. Dilatatio et hypertrophia atrii utriusque et ventriculi dextri cordis. Hydrops et cyanosis universalis. Aneurysma embolicum rami arteriae fossae Sylvii sin. Encephalomalacia et haemorrhagia lobi temporalis hemisphaerii sin. cerebri. Pneumonia Iobularis lobi inf. pulm. dextr.

Fall 9. O. P., 22 jähriger Konditor, hatte als Kind Masern und mit 10 Jahren schweren Gelenkrheumatismus, mit 17 Jahren Lungen- und Rippenfellentzündung. Seit 30. April 1905 ist er mit Schmerzen im rechten Knie und in der linken Schulter erkrankt.

Pat. zeigte vom 2.-18. Juni dauernd Temperaturen von $38^{\circ}$, hatte Gelenkschmerzen, dann fieberfrei und fast ohne Beschwerden. Am Herzen war eine Dilatation nach rechts, Verbreiterung des hebenden Spitzenstosses, lautes systolisches Geräusch und Klappen des 2. Pulmonaltones nachweisbar. Vom 9. Juli an traten Fiebertemperaturen bis $38,5^{\circ}$, starke Dyspnoe, Herzinsuffizienz und Beklemmungsgefühl auf. In der Nacht zum 18. Juli 
wurde Pat. sehr unruhig und klagte über heftige Kopfschmerzen, am folgenden Morgen Benommenheit, die dauernd tiefer wurde, und gegen Abend Exitus letalis.

Sektion (L.-Nr. 836/05; Obduzent: Herr Dr. Reinhardt):

Schädel symmetrisch, von glatter Aussen- und Innenfläche. Dura mater stark gespannt; die weichen Hirnhäute sind mässig blutreich und im $\mathrm{Be}-$ reich einiger später genannten Teile etwas gelbbräunlich pigmentiert. Die Windungen sind stark abgeflacht. Der rechte Schläfenlappen, der hintere, untere Abschnitt des Stirnlappens und der untere Teil des Scheitellappens wird durch einen beim Betasten weichen Herd eingenommen, über dem die Pia die erwähnte Verfärbung zeigt. Eine kleine, ebenso verfärbte Stelle findet sich vorne nahe der Mittellinie; doch ist hier die Hirnsubstanz selbst nicht verändert. Auf einem Horizontalschnitt kommt ein etwa faustgrosser mit dicken, dunkelroten Coagulis gefüllter Herd zum Vorschein, der sich in der weissen Substanz der früher erwähnten Lappen ausbreitet und sich besonders nach oben in das weisse Marklager erstreckt; nach innen reicht er bis an die grossen Ganglien. Die graue Substanz ist dabei nicht betroffen. Die Wand des Herdes besteht aus ziemlich wenig veränderter Hirnmasse, die etwas zerrissen, aber wenig erweicht ist und reichliche, kleine, rundliche Hämorrhagien aufweist. Offenbar ist die Hirnsubstanz durch den Bluterguss stark auseinandergedrängt. Beim Präparieren der Art. fossa Sylvii dextra kommt etwa $4 \mathrm{~cm}$ nach ihrem Abgange im Operculum ein etwas über bohnengrosser, hämorrhagischer Knoten zum Vorschein, der anscheinend aus geschichteten Thrombusmassen und zum Teil aus einer fibrösen Wand besteht und mit der Arterie zusammenhängt. Dieser Knoten liegt im Rande des grossen Herdes und zeigt nach diesem zu eine unregelmässig begrenzte Öffnung, die auch mit Gerinnseln gefüllt ist.

Sektionsdiagnose: Endocarditis chron. ulcerosa valv. mitralis. Dilatatio cordis. Aneurysmata embolica rami inf. arteriae mesentericae sup. et arteriae fossae Sylvii dextrae. Haemorrhagia permagna hemisphaerii dextri cerebri ex ruptura aneurysmatis art. fossae Sylvii dextrae. Infarctus veteriores embolici lienis et renis sin. Oedema pulmonum.

Fall 10. G. M., 42 jähriger Schlosser, hatte mit 29 Jahren schweren Gelenkrhenmatismus und behielt davon einen Herzfehler zurück. Seitdem mehrfach Residuen des Gelenkrheumatismus, zuletzt im Mai 1907 mit anfänglichem Schüttelfrost. Seitdem Nachtschweisse, häufige Anschwellung der Füsse und zeitweilige Sehstörung: "Nebelsehen".

Pat. wurde mit Schwellung und Schmerzhaftigkeit der Füsse am 7. Juni 1907 aufgenommen, hatte dauernd Temperaturen, die zwischen 37 und $38,5^{\circ}$ schwankten. Verbreiterung des Herzens nach rechts, undeutlicher Spitzenstoss, systolisches Geräusch an der Spitze und leises diastolisches Geräusch an der Aorta. Am 29. Juli wird Pat. unter dauernd zunehmender Schwäche benommen, am 30. Juli wird eine rechtsseitige schlaffe Lähmung und motorische Aphasie festgestellt. Dieser Zustand bleibt bis zum 6. August 1907, wo der Tod durch plötzlichen Kollaps eintritt, 
Sektion (L.-Nr. 1057/07; Obduzent: Herr Dr. Versé):

Schädeldach etwas länglich, gewölbt und ziemlich schwer: Die Dura liegt dem Gehirn glatt an; am vorderen Pole und an der Seite des linken Schläfenlappens befindet sich eine leichte, blutige Infiltration der weichen Häute. Die Art. carotis interna ist beiderseits frei, ebenso die Art. vertebrales und basilaris. Zwischen dem linken Stirn- und Schläfenlappen bestehen stärkere Adhäsionen, die blutig infiltriert sind, bei deren Lösung eine sackartige Erweiterung im mittleren Stamme der Art. cerebri media zum Vorschein kommt. Die Windungen sind auf dem linken Schläfenlappen etwas verbreitert; ihre Konsistenz an der Spitze des Lappens ist weich. Die weichen Häute auf der linken Hemisphäre sind leicht von Flüssigkeit durchtränkt, und beim weiteren Auseinanderziehen der Fossa Sylvii sin. zeigt sich, dass der Sack, in den die Art. cerebri media übergeht, mehr als erbsengross ist und eine etwas rauhe Fläche gegen den Stirnlappen hin besitzt, die von gelblich-rötlichen Gerinnselmassen gebildet wird. Die äbrige Oberfläche ist dagegen glatt. In der Tiefe erstreckt sich die offenbar von einer Perforation des Sackes ausgehende, blutige Infiltration weiter gegen die Insel.

Sektionsdiagnose: Endocarditis chron. fibrosa valvulae aortae. Insufficientia valvularum semilunarium aortae. Endocarditis ulcerosa gravissima valvulae mitralis, praecipue cuspidis anter. cum dilaceratione chordarum tendinearum. Endocarditis ulcerosa levis valvul. aortae. Hypertrophia et dilatatio cordis. Intumescentia septica et induratio cyanotica et abscessus metastaticus magnus lienis. Infarctus renum. Glomerulonephritis acuta. Aneurysma embolicum art. cerebri mediae sin. cum perforatione incipiente. Infiltratio haemorrhagica circumscripta leptomeningum fossae Sylvii sin. Oedema cerebri. Induratio cyanotica pulmon.

Fall 11. M. St., 37 jährige Arbeitersfrau, zog sich mit 18 Jahren durch Rheumatismus einen Herzfehler zu. Vom 2. XII. 1907 an hatte sie Frösteln, Fieber um $38^{\circ}$ und bot die Erscheinungen einer chronischulzerierenden Endocarditis; im Blute wurden spärliche Mengen von Streptococcus viridans nachgewiesen. Nachdem Pat. am 31. März 1908 die Klinik auf Wunsch verlassen hatte, kehrte sie am 11. Mai zurück, da sie dauernd sehr matt und elend gewesen war. An diesem Tage wurde sie plötzlich bewusstlos, zeigte Druckempfindlichkeit des Nackens, aber normalen Augenhintergrund. Am 15. Mai wurden reichliche Kolonien von Streptococcus viridans auf den Blutplatten nachgewiesen. Bis zum 27. Mai bellte sich das Bewusstsein völlig auf, und Pat. kann sich an die Zeit seit der Aufnahme in die Klinik nicht erinnern. Sie erholte sich jetzt gut, hatte nur zeitweilig leichte Temperatursteigerungen bis $38^{0}-38,3^{0}$; am Herzen war ein diastolisches Geräusch über der Aorta und Mitralis nachweisbar. Am 15. Juni ergab eine Lumbalpunktion klare Flüssigkeit obne Sediment. Am 28. Juni wurden wieder reichliche Mengen von Streptococcus viridans im Blute nachgewiesen. Am 27. Juli Bewusstlosigkeit mit Incontinenz; allmählich wurde Pat. etwas klarer, blieb aber völlig apathisch und liess Urin und Stuhl unter sich gehen, bis am 16. August der Tod eintrat. 
Sektion (L.-Nr. 1156/08; Obduzent: Herr Dr. Löhlein):

Schädeldach längsoval, symmetrisch, nicht adhäręnt. Dura mater ziemlich stark gespannt und zwar beiderseits gleichmässig; über dem vorderen Pol der rechten Hemisphäre scheint sie dunkelblänlich durch. Die Gefässe der Pia sind mässig stark gefüllt; in den Maschen der Pia im Bereich des rechten Stirnlappens, besonders in der Umgebung der Gefässe, findet sich eine reichliche, blutige Infiltration, so dass nur die zentralen Teile der Windungen sichtbar sind. Eine sehr massenhafte, teils flüssige, teils locker geronnene Blutansammlung findet sich weiter an der Basis des Gehirns, besonders in der Gegend des Infundibulum, der Abgänge der grossen Hirnnerven und als dickes Polster über der Unterfläche des Kleinhirns und der Medulla oblongata. Auch die Gegend des Foramen Monroi ist von einem bis zu Fünfmarkstückgrösse reichenden Bluterguss umgeben. Die Oberfläche des Kleinhirns dagegen ist frei von blutigen Infiltrationen der weichen Häute. Die Art. vertebrales und die Art. basilaris, sowie die Carotiden sind zartwandig, die Abgangsstelle der Art. profundae cerebri und der Art. communicans post. sind ehenfalls erkennbar und zart. Im Anfangsteil der Art. cerebri post. findet sich am vorderen Umfange ihrer Wand eine reiskorngrosse, gelbliche derbe Masse, in deren Grunde eine kleine sackförmige Ausbuchtung der Arterie erkennbar ist, die einen für eine feine Sonde durchgängigen, zackigen Riss ihrer Wand aufweist.

Sektionsdiagnose: Endocarditis chron, ulcerosa valvul. mitralis. Insufficientia valvulae. Endocarditis ulcerosa parietalis atrii sin. Dilatatio et hypertrophia ventriculi sin. cordis. Pericarditis chron. adhaesiva. Intumescentia lienis et hepatis. Infarctus embolici lienis. Nephritis chron, haemorrhagica (Glomerulonephritis). Infarctus veterior renis dextri. Aneurysma embolicum art. profund. cerebri dextr. rupt. Haemorrhagia baseos cerebri.

Diesen vier Fällen ist es gemeinsam, dass sich bei der Sektion eine ulzerierende Endokarditis des linken Herzens fand, die auch klinisch festgestellt oder vermutet worden war. Im Falle 10 und 11 konnte der Streptococcus viridans als Erreger der Sepsis nachgewiesen werden. Weiterhin zeigte sich in allen vier Fällen bei der Autopsie, dass der 'Tod durch Berstung eines cerebralen Aneurysma verursacht worden war. Die Entstehung dieser Gefässgeschwülste ist hier daher wohl mit Sicherheit auf die Embolie eines infektiösen Thrombus zurückzuführen, und dieser Annahme entspricht es auch, dass in dreien dieser Fälle die Art. cerebri media (Fossae Sylvii) befallen war, an der gerade embolische Aneurysmen unter den Hirnarterien so häufig sind.

Besonderes Interesse verdient bei diesen Fällen die Frage, ob es möglich ist, den Zeitpunkt der Embolie festzustellen, und m. E. ist dafür kein sicherer Anhaltspunkt vorhanden, Wohl aber lässt sich eine Vermutung aussprechen über die Entwicklungsdauer der Aneurysmen. Dieser Frage scheint bisher wenig Beachtung geschenkt wordeu zu sein, wenn sie auch schon von Lebert erörtert 
worden ist, und doch könnte sie für die Begutachtung eines Unfalles sehr wohl von Wichtigkeit werden. Wir wissen ja, dass gerade bei der Sepsis durch Streptococcus viridans Zeiten völliger Besserung und scheinbarer Heilung vorkommen, so dass die Kranken wieder ihre Arbeit aufnehmen können und damit Unfällen besonders leicht ausgesetzt sind.

Zur Beantwortung unserer Frage scheidet Fall 8 wegen unge- . nügender klinischer Daten ohne weiteres aus. In Fall 9 und 10 lag die erste Erkrankung an Gelenkrheumatismus und Endokarditis sehr lange, nämlich 12 oder 13 Jahre, zurück. Es ist schwerlich anzanehmen, dass damals die infektiöse Embolie der Hirnarterien stattgefunden hat. Der Beginn der eigentlich tötlichen Erkrankung fiel im Fall 9 in den Anfang Juni, der Tod durch Bersten des etwa bohnengrossen Aneurysma auf den 18. Juli; im Fall 10 traten die ersten Zeichen der Krankheit im Mai auf, während das mehr als erbsengrosse Aneurysma offenbar am 30. Juli, als die einseitige Lähmung: einsetzte, geplatzt ist. Es wäre also wohl in beiden Fällen höchstens eine Zeit von etwa 11/2 Monaten zur Entwicklung eines erbsen- bis bohnengrossen Aneurysma anzunehmen, so dass eine solche Gefässgeschwulst sich im Vergleich zu den sog. kongenitalen Aneurysmen ausserordentlich schnell vergrössert, was durch die zerstörende Einwirkung der Bakterien verständlich wird.

Im Fall 11 ist die Feststellung, wann die Embolie stattgefunden haben mag, sehr viel schwieriger, weil sich der Verlauf über ziemlich lange Zeit, nämlich über $3 / 4$ Jahre erstreckte. Und doch haben wir einen Anhaltspunkt, wann das Aneurysma innerhalb dieses Zeitraums schon vorhanden gewesen sein muss. Die Kranke wurde nämlich am 11. Mai plötzlich bewusstlos und zeigte Druckempfindlichkeit des Nackens. Diese Erseheinungen gingen bis zum 27. Mai völlig zurück, und es liegt der Gedanke nahe, dass am 11. Mai das Aneurysma schon zum ersten Male geplatzt ist. Von diesem Zeitpunkte an wird die Gefässgeschwulst schwerlich noch wesentlich gewachsen sein, weil sie nach einer einmaligen Perforation vermutlich bei weiterer Vergrösserung sofort wieder geplatzt wäre. Wir hätten also höchstens mit einer Entwicklungszeit von 1/2 Jahre, vermutlich aber mit einer kürzeren, zu rechnen. Immerhin muss das Aneurysma doch wohl 3 Monate (Mai bis Juli) bestanden haben und hat in dieser Zeit nur die Grösse eines Reiskornes erreicht, die im Vergleich mit den beiden anderen Fällen recht gering erscheint.

Durch diesen Fall (11) wird uns aber die weitere Frage nahegelegt, ob ein Hirnaneurysma entsprechend unserer obigen Annahme mehrere Male nacheinander zur Perforation gelangen kann. 
Zunächst erscheint es ja wahrscheinlicher, dass durch das Bersten eines solchen Gefässsackes, zumal wenn er im oder am Gehirn seinen Sitz hat, in kürzester Zeit der Tod herbeigefuihrt wird. Dennoch gibt uns zuweilen der Sektionsbefund Beweise dafür, dass mehrere zeitlich von einander getrennte Blutungen aus einem Aneurysma stattgefunden haben, wie es auf den nachstehenden Fall zutrifft:

Fall 12. M. T., 48jährige Bahnarbeitersfrau, litt längere Zeit schon an Unterleibsbeschwerden und hatte infolge einer Verletzung das rechte Auge eingebüsst. Anfang Januar 1908 erlitt sie einen Schlaganfall mit völliger Bewusstlosigkeit, Mitte Februar einen gleichen Anfall, und Anfang März wurde sie wiederum plötzlich bewusstlos. In diesem Zustande kam sie in die Klinik, wo bei der mittelkräftigen Frau deutliche Spasmen der rechtsseitigen Extremitäten mit Steigerung der Reflexe nachgewiesen werden konnten. Noch am gleichen Tage trat unter zunehmender Herzschwäche der Tod ein.

Sektion (L.-Nr. 358/08; Obduzent: Dr. Knierim II):

Schädeldach mässig dick, symmetrisch, seine Innenfläche glatt. Dura gespannt und an der linken Seite in der Gegend der Schläfenwindungen grünlich verfärbt. Die Sinus sind zum Teil ganz gefüllt, teils leer. Die Pia ist im Bereich des linken Schläfenlappens dunkelrot und blutig infiltriert, im übrigen ziemlich klar und durchsichtig. Auf einem Horizontalschnitt durch das Gehirn findet sich nahe der Oberfläche des linken Schläfenlappens eine reichlich walnussgrosse Höhle, die mit harten, braunroten Cruormassen angefüllt ist. Eine zweite, kleinere Blutung findet sich medial von der ersten und reicht bis zum hinteren Rande des Linsenkerns; hier befinden sich meist dunkelrote Cruormassen von weicher Konsistenz. Eine dritte Höhle, die mit dicken Blutgerinnseln angefült ist, befindet sich unmittelbar vor der ersten Höhle und ist von etwa gleicher Grösse, wie diese. Zwischen diesen beiden läuft eine Spange noch erhaltener, grauer Hirnsubstanz sichelartig von der Oberfläche in die Tiefe und erweist sich als Rindensubstanz des Schläfenlappens. Der Stirnlappen ist durch die Cruormassen vollkommen nach vorne gedrückt und verschoben. In der linken Fossa Sylvii befindet sich etwa $3 \mathrm{~cm}$ von ihrer Abgangsstelle eine kirschkerngrosse Ausbuchtung der Wandung der Art. cerebri media. Mit einer Sonde gelangt man leicht in den Sack hinein, aus dem man hinten oben wieder hinausgelangt. Die Öffnung hat die Grösse eines grösseren Stecknadelkopfes. Die Wandungen der Gehirnarterien sind ziemlich zart, nicht sklerotisch, überall gut durchgängig.

Sektionsdiagnose: Aneurysma arter. cerebri mediae sin. perforatum. Haemorrhagia magna veterior et recens in lobum temporal. sin. et nucleum lentiformem. Tuberculosis apic. pulm. dextri in sanatione et induratio nigra ibidem. Defectus bulbi oculi dextri. Polysaccia cordis. Perimetritis fibrosa. Hydrosalpinx bilateralis. Cystoma ovarii sin.

In der Krankengeschichte dieses Falles sind drei zeitlich von einander getrennte Schlaganfälle erwähnt, und die Obduktion liess ebenfalls drei von einander abgrenzbare Blutungsherde erkennen. Als 
Ursprung der Blutungen kam einzig und allein das Aneurysma der linken Art. cerebri media in Frage, da ja an den übrigen Hirnarterien nichts krankhaftes gefunden wurde und die Blutungsherde in der Nähe der Aneurysma zusammenstiessen. Dieses Beispiel, dem sich übrigens der früher erwähnte Fall 6 anreibt, lässt keinen $Z$ weifel darüber aufkommen, dass tatsächlich ein einmal perforiertes Hirnaneurysma sich wieder schliessen und später noch ein oder mehrere Male zu einer Blutung führen kann. In gleicher Weise dürfte daher auch wohl die nachstehende Krankengeschichte zu deuten sein, zumal der Sektionsbefund deutlich das Vorhandensein älterer Thrombusmassen an der Gefässausbuchtung nachwies.

Fall 13. H. H., 57 jährige Witwe, ist angeblich seit 4 Wochen selır matt gewesen, brach dann am 30. Juni plötzlich zusammen und wurde bewusstlos in die Klinik eingeliefert. Hier konnte links eine schlaffe Lähmung des Armes und positiver Babinskischer Reflex nachgewiesen werden. Die Bewusstlosigkeit ging bald in leichte Benommenheit über, und die Kranke erholte sich leidlich, bis plötzlich am 7. Juli von nevem ein tiefes Coma mit deutlicher Pulsverlangsamung einsetzte. Pat. starb wenige Stunden darauf.

Sektion (L.-Nr. 808/06; Sektionskurs):

Schädeldach symmetrisch, flach, zeigt innen tiefe Gefässfurchen. Dura mater ziemlich prall gespannt, rechts stärker als links. Die Windungen der linken Hemisphäre sind abgeplattet, die Gefässe ziemlich stark injiziert. Auf der rechten Seite findet sich in der Umgebung der Fossa Sylvii eine ziemlich grosse, blutig infiltrierte Stelle von mehr als Fünfmarkstückgrösse. Auf einem horizontalen Durchschnitt sieht man auf der rechten Seite, die schon von aussen ansgedehnt erscheint - besonders in der Gegend der Insel - ein etwa hühnereigrosses Coagulum, das besonders den Nucleus caudatus and die Capsula externa einnimmt, sich aber bis zum äusseren Rande des Thalamus hinzieht. Die innere Kapsel ist stark gequollen, der äussere Rand des Nucleus lentiformis, der in die Blutung übergeht, erweicht. Die weichen Häute sind über diesem Blutcoagulum, besonders in der Inselgegend blutig unterlaufen. Die Arterien der Basis sind zartwandig. Beim Herauspräparieren der rechten Art. fossae Sylvii zeigt sich, dass zwischen Schläfen- and Stirnlappen in dem dort vorhandenen grossen Blutgerinnsel an einem Aste der Art. cerebri media ein etwa erbsengrosser, dunkelrot gefärbter, dickwandiger Sack hängt, vor dem sich eine etwa hanfkorngrosse Ausbuchtung der Arterienwand findet. Man gelangt mit einer feinen Sonde von dem Hauptstamme der Arterie aus in diese aneurysmatische Erweiterung. Die Fortsetzung der Arterie jenseits dieser Ausbuchtung ist nicht zu sondieren. Die Blutung hat sich von hier aus hauptsächlich gegen die Insel $\mathrm{zu}$ in den weichen Häuten verbreitet und ist auch hier in die Gehirnmasse selbst eingedrungen. Nach dem Herauslösen der Arterie aus diesen Blutmassen zeigt sich, dass das vorhin erwähnte, erbsengrosse, sackartige Gebilde im wesentlichen offenbar aus Thrombusmassen zusammengesetzt ist, die der ganz dünnwandigen, hanfkorngrossen Ausbuchtung der Gefässwand anhängen. Ein weiterer kleiner knopfartiger Vorsprung wölbt sich am 
Eingang zu der zartwandigen Ektasie stärker nach aussen vor. Diese Ausbuchtung sitzt gerade im ersten Teilungswinkel der Arterie zwischen den auseinanderweichenden Hauptästen.

Sektionsdiagnose: Aneurysma circumscriptum perforatum rami art. cerebri mediae dextrae. Infiltratio haemorrhagica leptomeningum lateris dextri, praecipue fossae Sylvii. Infiltratio haemorrhagica insulae, capsulae externae, nuclei lentiformis dextri. Atheroscerosis mediocris aortae. Kyphosis dorsalis. Tuberculosis obsoleta apic. pulm. sin. Taenia mediocanallata. Ephelides faciei et antibrachii utriusque.

Mit Hilfe des Obduktionsbefundes gelingt es also zuweilen, das Auftreten mehrerer Blutungen aus einem Aneurysma nacheinander tatsächlich festzustellen. Zur Ergänzung unserer Beobachtungen mögen hier noch kurz die ron Beykovsky, Rosenberg (Fall 1), Möser (Fall 1), Me Nalty und Pfeufer berichteten Fälle genannt sein. Meist wird es, wie bei allen diesen Beispielen, möglich sein, aus der Krankengeschichte dén Zeitpunkt der einzelnen Blutungen festzustellen, indem plötzlich schwerere klinische Erscheinungen beim Eintritt der Hämorrhagie einsetzten.

Glückt es uns so, bei vorliegendem Sektionsergebnis zurückschauend, bestimmte Ereignisse im klinischem Verlaufe als durch aneurysmatische Blutung bedingt zu erkennen, so soll damit keineswegs gesagt sein, dass nun auch umgekehrt der Kliniker aus dem Vorhandensein jener Symptome mit einiger Sicherheit schon auf das Vorhandensein eines geplatzten Aneurysma schliessen darf. Der Eintritt eines apoplektiformen Insults, wie er in unseren Fällen 12 und 13 das Auftreten der einzelnen Blutungen bezeichnete, ist sicherlich ein klinisch allzu vieldeutiges Symptom, als dass es für aneurysmatische Blutungen irgendwie charakteristisch sein könnte.

Es wäre jedoch von grossem Werte, wenn wir wenigstens eine klinische Erscheinung wüssten, die schon intra vitam unseren Verdacht gerade auf die aus Hirnaneurysmen stammenden Blutungen lenken würde. Ein solches Symptom, das allerdings nur mit Berücksichtigung aller übrigen Erscheinungen und des ganzen klinischen Verlaufs zu verwerten ist, ist tatsächlich zuweilen vorhanden und besteht in dem Auftreten von Nackenstarre. Es ist eine durch reichliche Erfahrung festgestellte Tatsache, dass, wie wir früher schon einmal kurz erwähnten, fast sämtliche Hirnaneurysmen an der äusseren Oberfläche des Gehirns sitzen. Sie werden daher im Falle einer Ruptur durchweg meningeale Blutungen anstatt solcher in das Gehirnparenchym allein erzeugen, und das ergossene Blut fliesst dann nach hinten zu in den Rückenmarkskanal. Die dadurch hervorgerufene Reizung der Meningen in der hinteren Schädelgrube und im oberen 
Teile des Spinalkanals bedingt offenbar das Auftreten der Nackenstarre, zu der zuweilen eine Steifheit der ganzen Wirbelsäule binzutreten kann. Dieses Symptom war im nachstehenden Falle sehr ausgesprochen vorhanden, so dass er hier eingeschaltet sein mag:

Fall 14. M. Sch., 36jährige Frau, im 6. Monat gravid, ist bisher gesund gewesen. Am 15. Juli 1905 erkrankte sie plötzlich an heftigen Kopfschmerzen und vermochte den Kopf nicht mehr nach vorn zu beugen. Fieber, Erbrechen und Krämpfe fehlten. Bei der Aufnahme in die Klinik am 16. Juli konnte bei der kräftigen und gut genährten Kranken, deren Bewusstsein bis dahin und auch jetzt nicht getrübt war, als einziges, objektives Symptom eine deutlich ausgeprägte Nackenstarre nachgewiesen werden. Der Kopf schmerzte in geringem Maße schon bei völliger Ruhelage, jede Bewegung war aber äusserst schmerzhaft und liess sich daher kaum ausführen. Die Pulszahl betrug 96, die Temperatur 36,6 ${ }^{\circ}$. Während des Tages steigerten sich die Kopfschmerzen immer mehr, allmählich wurde Pat. unruhiger und fing an zu delirieren. Abends trat plötzlich innerhalb weniger Minuten der Tod durch völligen Atemstillstand ein.

Sektion (L.-Nr. 829/05; Obduzent: Herr Dr. Reinhardt):

Schädel sehr lang und schmal, symmetrisch. Dura mater gespannt, ihre Innenfläche trocken. Weiche Hirnhäute mässig bluthaltig. Die Hirnwindungen sind überall abgeflacht und ziemlich breit, die Furchen verstrichen. An der Basis findet sich im Bereiche des Infundibulums, des Pons, der Medulla obglongata und weiterhin, von hier auf die Unterfläche des Kleinhirns, auf die Tonsillen und den hinteren Umfang des Kleinhirns übergehend, eine mehrere Millimeter starke, blutige Infiltration der weichen Hăute. Das Blut ist dick geronnen, und der Bluterguss ist am stärksten an beiden Tonsillen des Kleinhirns, wo er links sogar eine Dicke von $1 \mathrm{~cm}$ erreicht. Am medialen Rande der linken Tonsille haftet der Bluterguss etwas fester an und zwar an einem etwas über stecknadelkopfgrossen, dunkelroten Kä̈tchen, das mit der Art. cerebelli post. inf. zusammenhängt und ein kleines geplatztes Aneurysma darstellt. Der Bluterguss erstreckt sich vom Kleinhirn durch das Foramen Magendii in den 4. Ventrikel, der dadurch ganz ausgefüllt wird. Das Coagulum reicht weiter durch den Aquaeductus Sylvii in den 3. Ventrikel, der ebenfalls ausgefüllt ist, und ragt beiderseits mit einem etwa $3 \mathrm{~cm}$ langen Fortsatz in den Seitenventrikel hinein. Die Seitenventrikel sind etwas erweitert und enthalten reichlich blassrötliche, klare Flüssigkeit, deren Retention offenbar durch Verlegung des Foramen zur Erzeugung der Hirnanschwellung mit beigetragen hat. In der Schädelbasis sammelt sich bei Herausnahme des Gehirns reichliche, blutige Flüssigkeit an; ebenso fliesst von den weichen Häuten der Basis etwas flüssiges Blut ab und auch aus dem Spinalkanal entleert sich reichlich blutige Flüssigkeit.

Die Hirnsubstanz ist ziemlich feucht, mässig blutreich, von mittlerer Konsistenz, ohne besondere Herde. Nur die Substanz der Medulla oblongata ist im Bereiche des Foramen Magendii sehr weich, offenbar infolge des von oben auf sie einwirkenden Druckes des Blutergusses. Die Substanz der Tonsillen ist ebenfalls etwas weich. Die Hirnarterien sind sämtlich dünnwandig, zart und durchscheinend, was auch auf die zu den Knötchen hinführende Art. cerebelli zutrifft. Das Knötchen zeigt auf Serienschnitten 
ein kleines Aneurysma, dessen Wand stark verdünnt, an einer Stelle eingerissen und aussen mit Thrombusmassen belegt ist. Diese letzteren, deren innerer Teil etwas älter ist, sind von der Wand an einer Seite abgelöst.

Sektionsdiagnose: Ruptura aneurysmatis parvi arteriae cerebelli post. inf. sin. Haemorrhagia intrameningealis cerebelli, baseos cerebri medullae et intraventricularis. Malacia medullae oblongata compressione haematomatis effecta. Endocarditis verrucosa incipiens valvulae mitralis. Oedema pulm. Synostosis suturarium cranii. Dolichocephalia. Graviditas mensis VI.

Dieser Fall zeigt uns mit besonderer Deutlichkeit, dass eine zunächst offenbar nicht allzu schwere Blutung in die Meningen der hinteren Schädelgrube ausgesprochene Nackensteifigkeit hervorruft. Das Bewusstsein blieb hier, was ja meist nicht der Fall sein wird, völlig erhalten; es fehlten neben der Nackenstarre alle objektiven Erscheinungen, und subjektiv wurde nur über heftigen, zunehmenden Kopfschmerz geklagt. Der Verlauf endlich war in diesem Falle ausserordentlich rasch, weil sehr bald eine neue Blutung den Tod herbeiführte. Ein ähnliches, interessantes Beispiel von mehr protrahiertem Verlauf bietet folgende Beobachtung:

Fall 15. W. R., 42jährige Näherin, litt seit vielen Jahren an heftigem Druckgefühl im Kopfe, das anfallsweise alle 8-14 Tage auftrat und mehrere Tage anzuhalten pflegte. Etwa vier Wochen vor der Aufnahme in die Klinik hatte sie wieder heftige Kopfschmerzen, fühlte sich sehr unwohl und bekam Brustbeklemmungen. Sie setzte sich daher auf ein Sofa und wurde dort bald darauf bewusstlos gefunden. Dieser Zustand hielt mehrere Tage an, und erst allmählich erholte sich die Kranke, um zunächst einige Tage zwar schon selbst etwas sprechen, aber Gesprochenes noch nicht verstehen zu können. Nach völliger Rückkehr des Bewusstseins war sie noch sehr matt. Annähernd 3 Wochen nach dem ersten Anfalle trat wiederum ein solcher mit äusserst heftigem Kopfschmerz und grosser Schwäche, aber nur mit leichter Benommenheit auf, weshalb Pat. in die Klinik gebracht wurde. Es war ferner aufgefallen, dass bei der Pat. im Laufe dieser letzten Wochen das Sehvermögen auf dem linken Auge sich dentlich verschlechtert hatte.

Bei der ziemlich kräftigen Kranken fiel sofort der starre, etwas leidende Gesichtsausdruck auf. Die Kranke machte einen leicht apathischen Eindruck. Der Kopf war im Nacken nach hinten überstreckt und wurde unbeweglich gehalten. Pat. gab selbst an, dass der Nacken steif sei; es gelang auch nicht, ihn nach vorn zu beugen, während eine seitliche Drehbewegung leicht auszuführen war. Die Augenbewegungen waren normal, die rechte Pupille war weiter als die linke, beide reagierten träge auf Licht und Akkommodation. Der Geruchssinn erschien links herabgesetzt. Über der rechten Lunge waren kleinblasige Rasselgeräusche hörbar. Das Befinden änderte sich bis zum Abend des folgenden Tages wenig; um diese Zeit setzte plötzlich eine schwere Bewusstlosigkeit ein, und der Kopf wurde jetzt noch mehr nach hinten überstreckt. Die Atmung verlangsamte sich 
stark, der vorher immer leicht beschleunigte Puls nahm eine Frequenz von 144 in der Minute an. Alle Reflexe waren erloschen, und etwa $1 / 4$ Stunde nach Beginn dieser Erscheinungen trat der Tod ein.

Sektion (L.-Nr. 769/85; Obduzent: Herr Dr. Huber):

Schädeldach von mittlerer Dicke; die Dura liegt der Gehirnsubstanz innig an, die weichen Häute sind an Konvexität milchig verdickt. An der Basis sind sie von reichlichen Mengen schwarzroten, geronnenen Blutes prall angefüllt, und zwar besonders stark im Anfangsteil der Fossae Sylvii, zwischen dem Chiasma und Pons. Die Art. carotis sin. ist unmittelbar vor der Einmündung in den Circulus arteriosus in einen rundlichen, kirschgrossen Sack verwandelt, der in seinem Innern mit schwarzrotem Blutgerinnsel prall gefüllt ist und an seiner oberen, der Hirnbasis zugekehrten Fläche in der Richtung gegen die Frontalspalte eine für eine dünne Sonde gerade noch durchgängige Öffnung zeigt. Diese führt aus dem Innern des Sackes unmittelbar in die weichen Hirnhäute und zu den hier befindlichen Blutgerinnseln. Die Ränder des Sackes sind an dieser Stelle sehr verdünnt; an den übrigen Teilen zeigt sich die Wand von gleicher Dicke, wie das gewöhnliche Arterienrohr, und es finden sich keinerlei sklerotische Prozesse in der Wand des Sackes. Auch in den angrenzenden Hirnhäuten ist keine Verdickung oder Schwielenbildung nachweisbar. Das Aneurysma geht unmittelbar, scharf abschneidend, in das Arterienrobr über, welches an der Grenze seine normale Weite zeigt und gleichfalls keinerlei Verdickungen aufweist. Auch die übrigen Arterien der Basis lassen nur hier und da ganz geringe Verdickungen erkennen, die keine besonderen Merkmale an sich tragen. Weitere Aneurysmen lassen sich nicht wahrnehmen. Der linke Nervus opticus ist in seiner ganzen Länge vom Austritt aus dem Traktus bis zum Durchtritt durch die Basis stark platt gedrückt und mit dem Aneurysma einseitig verwachsen. Dasselbe gilt von den angrenzenden Teilen der linken Hälfte des Chiasma. Der linke Tractus olfactorius ist in seiner hinteren Hälfte und an der Stelle, wo er aus der Hirnsubstanz heraustritt, durch das Aneurysma stark nach links ausgebuchtet und plattgedrückt. Die Hirnbasis zeigt einen der Grösse und Lage des Aneurysma entsprechenden Eindruck von rundlicher Gestalt. Die übrigen Hirnnerven sind intakt. Die Gehirnsubstanz ist weich, von wenig Blutpunkten durchsetzt und zeigt keine Besonderheiten.

Sektionsdiagnose: Aneurysma magnum perforatum arteriae carotidis int. sin. nervum opticum sin., tractum opticum sin., partem post. tractus olfactorii sin. comprimens. Infiltratio haemorrhagica diffusa leptomeningum baseos cerebri. Arteriosclerosis levis aortae et art. femoralis. Cyanosis universalis.

Forschen wir in diesem Falle zunächst nach der Ätiologie des Aneurysma, so gibt uns die Krankengeschichte dafür insofern einen Fingerzeig, als der erste Anfall von Bewusstlosigkeit wohl schon bestimmt mit der Gefässgeschwulst in ursächlichem Zusammenhang zu bringen ist. Da diese aber offenbar von einem der seit Jahren wiederkehrenden Anfälle von Kopfdruck oder -Schmerz eingeleitet wurde, erscheint die Vermutung berechtigt, auch diese auf das Aneurysma zurückzuführen. Die Sektion zeigte nun ferner, dass weder syphili- 
tische noch sklerotische Veränderungen vorhanden waren, und dass das Arterienrohr zu beiden Seiten des Aneurysma frei durchgängig, also nicht durch eine Embolie verlegt war, auf die sonst die erste $\mathrm{Be}$ wusstlosigkeit zurückgeführt werden könnte; infolgedessen sind wir wohl berechtigt, bei der 42jährigen Kranken ein Aneurysma auf kongenitaler Anlage anzunehmen, und die Frage nach der Ursache des ersten Anfalles von Bewusstlosigkeit beschränkt sich bei dem Mangel aller sonstiger Hirnveränderungen auf die Entscheidung, ob dieses Ereignis durch eine plötzliche Vergrösserung des Aneurysma und schon durch eine Blutung aus diesem hervorgerufen wurde.

Eine plötzliche Erhöhung des Blutdrucks kann ja zweifellos einen an und für sich schon dünnen Gefässsack erheblich ausdehnen und gerade bei Hirnaneurysmen durch Kompression benachbarter, funktionell wichtiger Teile deutliche klinische Erscheinungen hervorrufen. So erwähnt Oppenheim in seinem bekannten Lehrbuche der Nervenkrankheiten (I, S. 1064) einen Fall, bei dem im Anschluss an einen starken Hustenanfall die Erscheinungen eines Aneurysma der Art. profunda cerebri auftraten. Im allgemeinen wird es sich jedoch dabei wohl meist nur um Herderscheinungen handeln, und daher ist es gerade in unserem Falle wenig wahrscheinlich, dass die tagelang bestehende Bewusstlosigkeit nur auf eine Volumenzunahme des in der vorderen Schüdelgrube gelegenen Aneurysma zurückzuführen war. Demgegenüber erklärt sich dieser Zustand aber sehr wohl durch die Annahme einer Perforation der Gefässgeschwulst und einer damit verbundenen Blutung, und wir gelangen so hier zunächst nur per exclusionem auf Grund des Sektionsbefundes zu der Annahme einer erstmaligen aneurysmatischen Blutung.

Diese Auffassung findet nun weiterhin eine gewisse Bestätigung durch die bei dem zweiten Anfalle beobachteten Erscheinungen. Wieder begann dieser mit ausserordentlich heftigen Kopfschmerzen neben allgemeinem Schwächegefühl, ohne dass es dieses Mal dabei zu völligem Verlust des Bewusstseins kam. Bei der kurz darauf erfolgten Aufnahme in die Klinik wurde nun festgestellt, dass die Kranke den Kopf unbeweglich nach hinten gestreckt hielt und selbst über Nackenstarre klagte, so dass sie den Kopf nicht vorwärts beugen konnte. Die gleiche Erscheinung trat in verstärktem Maße bei dem dritten Anfalle auf, der unter schwerster Bewusstlosigkeit schnell zum Tode führte. Die Nackenstarre kann auch in diesem Falle, da sie ja sonst mit cerebralen Begleiterscheinungen wohl nur bei Affektionen der hinteren Schädelgrube und bei basaler Meningitis vorkommt, einzig und allein nur durch den Bluterguss in die weichen Hirnhäute bis zum Pons hin erklärt werden.

Deutsche Zcitschrift f. Nervenheilkunde. Bd. 44. 
Das Symptom der Nackensteifigkeit scheint daher bei cerebralen Aneurysmen überhaupt nicht so ganz selten zu sein, und dürfte bisher wohl für die Diagnostik dieses Leidens zu wenig benutzt worden sein. Bei der Durchsicht der mir im Original zugänglichen Literatur fand ich unter 74 klinisch eingehend beschriebenen Fällen, bei denen die Sektion ein Hirnaneurysma ergab, nicht weniger als 23 Fälle (= 31 Proz.), in denen diese Erscheinung besonders hervorgehoben war, und doch war in fast allen diesen Fällen an die Möglichkeit eines Hirnaneurysma bei der Diagnose gar nicht gedacht worden soweit das wenigstens aus den Veröffentlichungen selbst zu ersehen ist.

Ob gelegentlich schon durch den Sitz der Gefässerweiterung in der hinteren Schädelgrube allein Nackensteifigkeit hervorgerufen werden kann, lässt sich nach der mir vorliegenden Literatur nicht entscheiden. Die Möglichkeit wird aber wohl zugegeben sein; damit wird jedoch die Bedeutung des Symptoms für die Erkennung meningealer und besonders aneurysmatischer Blutungen nicht herabgemindert. Denn nicht nur das Symptom als solches, sondern die Art seines Auftretens ist charakteristisch. Während bei Meningitiden die Nackenstarre allmählich einsetzt und zunimmt, tritt sie hier plötzlich in die Erscheinung oder wird wenigstens unmittelbar im Anschluss an eine plötzliche Bewusstlosigkeit bemerkbar.

Gerade das Auftreten von Nackenstarre unmittelbar nach einem apoplektiformen Insult muss deshalb schon am Lebenden den Gedanken an ein geplatztes, cerebrales Aneurysma wachrufen. Es wird dann neben der Feststellung anderer Symptome oder besonderer ätiologischer Momente, die für eine solche Gefässgeschwulst sprechen, vor allem darauf ankommen, das Vorhandensein einer meningealen Blutung einwandfrei nachzuweisen. Das einzig sichere Mittel zu diesem Zwecke ist die Lumbalpunktion, und sie wird uns deshalb für die Diagnostik der Hirnaneurysmen von grösstem Wert sein (vgl. Rosen berg).

Mit Recht ist allerdings auf die Gefahr eines solchen Eingriffes aufmerksam gemacht worden, da durch eine schnelle und beträchtliche Druckerniedrigung im Lumbalkanal eine neue Blutung herrorgerufen werden kann. Diese Bedenken dürften aber nicht mehr zutreffen, wenn man unter Verzicht auf die Druckmessung nur tropfenweise aus einer mit Hahn versehenen Kanüle den Liquor abfliessen lässt, wobei es sich empfiehlt, 2-3 Proben von je 1 bis höchstens $2 \mathrm{ecm}$ zu entnehmen. Da es sich sehr häufig um benommene und apathische Kranke handelt, hat man auch eine erhebliche Blutdrucksteigerung infolge der vielleicht mit dem Eingriff verbundenen Erregung nicht allzu sehr zu befürchten oder sorgt dafür, ihr durch 
Beruhigungsmittel vorzubeugen. In den meisten Fällen wird es sich ja auch zunächst nur um die Vermutung einer meningealen. Blutung handeln, und eine Lumbalpunktion wird zur Sicherstellung der Diagnose zwecks Einleitung einer wirksamen Behandlung dringend wünschenswert sein.

Bei der Vornahme dieses Eingriffs ist es allerdings erforderlich, die Technik völlig zu beherrsehen und die Veränderungen, die das Blut innerhalb des Lumbalkanals einzugehen pflegt, genau zu kennen. Es sei daher an dieser Stelle kurz auf die Arbeiten von Tuffier, Milian, Ohm, Reichmann und anderer verwiesen. Dass dann unter Berücksichtigung aller klinischen Symptome die sorgfältige Prüfung des Liquor cerebrospinalis für die Diagnose der cerebralen Aneurysmen einen wesentlichen Fortschritt bedeutet, zeigen schon die von Nothnagel, Rindfleisch, Ohm und Rosenberg veröffentlichten Fälle. Ihnen ähneln in vieler Beziehung drei weitere Beobachtungen aus der Leipziger medizinischen Klinik, über die schon vor kurzem einmal gelegentlich eines Vortrages in der Leipziger medizinischen Gesellschaft berichtet wurde.1) Da sie jedoch in diesem Zusammenhange zur Abrundung der hier gegebenen klinischen Darstellung wesentlich beitragen dürften, sei ihre nochmalige Mitteilung unter Hinzufügung der ausführlichen Sektionsprotokolle gestattet.

Fall 16. H. G., 42jähriger Arbeiter, wurde am 23. Februar 1909 bewusstlos in die Klinik eingeliefert. Die später erhobene Anamnese ergab, dass er ausser Masern und Scharlach noch mit 22 Jahren einige Zeit Husten und Auswurf gehabt und sich mit 24 Jahren syphilitisch infiziert hatte. Seit 2 Monaten litt er an zeitweiliger Nackensteifigkeit und Kopfschmerzen. Am Tage der Einlieferung stiess er morgens mit dem Schienbein gegen einen Karren, fiel bald darauf bewusstlos um und wurde deshalb in die Klinik geschafft.

Der Aufnahmebefund (23. II.) ergab tiefes Coma, fehlende Pupillenreaktion, Nackenstarre, kein Fieber, 80 Pulsschläge und auf der rechten Lungenspitze eine leichte Schallverkürzung.

24. II. Sensorium klare:, starke Nackensteifigkeit, sehr heftige Kopfschmerzen, Pupillen reagieren; Lumbalpunktion ergibt $2 \mathrm{ccm}$ blutigen Liquors mit deutlicher Vermehrung der Lymphocyten.

In den folgenden Tagen vollständige Räckkehr des Bewusstseins, aber dauernd heftige Kopfschmerzen und Nackenstarre, kein Fieber, keine Hirndrucksymptome.

Am 26. II. und am 3. III. wurde nochmals je eine Lumbalpunktion vorgenommen, die stets bluthaltigen Liquor mit Lymphocytenvermehrung ergab.

12. III. Plötzlicher Aufschrei, dann allgemeine Krämpfe und Bewusstlosigkeit. Nach einigen Stunden zweiter Krampfanfall und

1) S. Wichern, Münch. med. Wochenschrift 1911. Nr. 51. 
seitdem deutliche Vermehrung der inzwischen etwas geringer gewordenen Nackensteifigkeit.

In den folgenden Tagen allmähliche Besserung; Sensorium dauernd leicht benommen, aber Nahrungsaufnahme per os nicht gestört. Trotzdem zunehmender Kräfteverfall. Nackenstarre unverändert stark.

23. III. Plötzlicher Verfall und Tod.

Sektion (L.-Nr. 422/09; Obduzent: Herr Privatdozent Dr. Versé):

Schädeldach lang, an der Innenfläche rauh. An der linken Seite finden sich in der Gegend des Stirnlappens flache Verkalkungen der Dura; diese selbst ist gespannt und zeigt ziemlich stark gefüllte Gefässe. Die weichen Hirnhäute sind zart, trocken, teilweise hellgelb durchscheinend. Die Gehirnwindungen sind platt. Bei der Herausnahme des Gehirns zeigt sich, dass die Hypophyse nicht besonders stark abgeplattet ist; auch das Infundibulum ist nicht sehr ausgedehnt. Das Gehirn selbst hat eine feste Konsistenz; an der Basis findet sich eine ausgedehnte blutige Infiltration der weichen Häute, die bis zum Stiel der Hypophyse und hinten unten bis an das Kleinhirn heranreicht. Die Gehirnnerven sind von diesen Blutmassen völlig umgeben; nach abwärts setzt sich die blutige Infiltration unter der Arachnoidea in den Spinalkanal fort. An der Unterfläche der Medulla oblongata findet sich links eine leichte Vorwölbung und eine mehr grau aussehende Zeichnung. Die Art. vertebrales erscheinen auf dem Durchschnitt etwas verdickt, ebenso die Art. cerebri mediae. Nach der Härtung wird das Gehirn durchschnitten. Neben der Art. vertebralis sin. erscheint ein erbsengrosser Sack, der von einer bindegewebigen Wand gebildet wird. Die Infiltration der weichen Häute mit Blut ist an dieser Stelle etwa $81 / 2 \mathrm{~mm}$ stark. Die linke Hälfte der Medulla oblongata ist stark zusammengepresst, namentlich die Pyramidenbahn, aber auch die Olive. Die Blutung geht auch auf die Substanz der Medulla uber. Sowohl der vierte Ventrikel, als auch die Seitenventrikel sind mit mässigen Mengen Blutes gefüllt. Nach Eröffnung des Spinalkanals scheint der Inhalt des Duralsackes blau durch; besonders unten ist die Dura ziemlich stark gespannt und ausgedehnt, aber auch oben im Halsteil ist die Spannung ziemlich beträchtlich. Beim Durchschneiden des Duralsackes. an der Cauda entleert sich reichlich blutig gefärbte Flüssigkeit. Nach Aufschneiden der Dura zeigt sich, dass der ganze Subarachnoidalsack mit. geronnenen Blutmassen angefüllt ist.

Sektionsdiagnose: Aneurysma perforatum art. vertebral. sin. Infiltratio haemorrhagica gravis diffusa leptomeningum baseos cerebri et medullae spinalis. Contenta sanguinolenta ventriculorum cerebri. Dilatatio vesicae urinariae. Bronchitis purulenta.

Fall 17. E. K., 54jähriger Werkmeister, der 1909 an einem Furunkel im Nacken gelitten hatte und sonst gesund gewesen war, klagte seit Sommer 1910 über hänfig auftretende Kopfschmerzen und Schwindelgefühl ohne Erbrechen. Am 29. Jan. 1911 fiel er nach einer Aufregung plotzlich vom Sofa, erbrach und wurde bewusstlos.

Bei der sofortigen Einlieferung in die Klinik (29. I.) war er noch somnolent, warf sich unruhig umher und griff stöhnend nach dem Kopfe. Kein Fieber. Puls 88. Sofortige Lumbalpunktion ergab $8 \mathrm{ccm}$ rein blutigen Liquors mit 800000 Erythrocyten im cbmm und $20 \%$ Eiweiss. 
Das Blut gerann nicht, die Farbe des Liquor selbst war "schwach gelb. Am Augenhintergrund fanden sich beiderseits einige kleine Netzhautblutungen.

30. I. Sensorium klarer, heftige Kopfschmerzen, mässige Nackenstarre, Kernigsches Symptom positiv. Urin enthält Spuren Eiweiss.

31. I. Geringe Besserung. Lumbalpunktion ergab $6 \mathrm{ccm}$ blutigen, nicht gerinnenden Liquors mit $21 \% 2 \%$ Eiweiss und deutlicher Xanthochromie.

2. II. Gestern Anstieg der Temperatur auf $37,8^{\circ}$, heute vermehrter Kopfschmerz, Nacken sehr druckemptindlich. Kernig +, motorische Reflexe normal.

3. II. Zustand unverändert. Lumbalpunktion: $3 \mathrm{ccm}$ mässig blutigen Liquors mit 38000 Erythrocyten im cbmm, 1\%o Eiweiss und Xanthochromie.

5. II. Morgens plötzlich heftigster Kopfschmerz und stechender Schmerz im rechten Unterkiefer, nach 1/4 Stunde zunehmende Benommenheit, Puppillen sehr eng, rechte Nasolabialfalte verstrichen, maximaler Opisthotonus. Völliges Aussetzen der Atmung, nach 5 Minuten Exitus letalis.

Sektion (L.-Nr. 218/1911; Obduzent: Herr Privatdozent Dr. Versé):

Schädeldach länglich, symmetrisch, fest an der Dura haftend. Die weichen Hüute an der Basis sind ganz mit Blut infiltriert, das sich durch die Fossa Sylvii fast bis an die Kante der beiden Scheitellappen erstreckt. Die rechte Kleinhirnhemisphäre ist ausgedehnt; an ihrer oberen Fläche weicht besonders nach vorne hin die Rindensubstanz auseinander, und in der Tiefe erscheinen dunkelrote, coagulierte Blutmassen. Nach abwärts setzt sich dic Infiltration unter der Arachnoidea in den Spinalkanal fort. Die Cisterna subcerebellaris ist ganz von den geronnenen Blutmassen bedeckt, die unter der Arachnoidea liegen. Die Art. basilaris und die Art. vertebrales sind weit, glatt, ihre Wand dick, aber nicht stark verändert. Die Art. carotides zeigen leichte gelbliche Verdickungen. An der Unterfläche und seitlichen Kante des linken Schläfenlappens sind etwa fünfpfennigstückgrosse, oberflächliche Substanzverluste mit dunkelbrauner Verfärbung sichtbar. Auf dem Durchschnitt durch Pons und Kleinhirn findet sich in der rechten Kleinhirnhemisphäre ein länglich-ovaler, meist aus geronnenen Blutmassen bestehender Erguss von $6 \mathrm{~cm}$ Länge, $3,5 \mathrm{~cm}$ Breite, der am Nervus trigeminus durch die Rindensubstanz in die weichen Häute durchgebrochen ist. Auch der vierte Ventrikel ist mit Blut gefüllt. Der vordere Teil des Blutergusses ist etwas fester geronnen, als der hintere Abschnitt. Die Häute des Rückenmarks sind blutig verfärbt; stellenweise finden sich darin auch einige grössere dunkle Gerinnsel. In die Arachnoidea sind vielfach zackige, zum Teil linsengrosse Kalkplatten eingelagert.

Sektionsdiagnose: Sclerosis arteriarum parv. renum. Nephritis interstitialis chron. Hypertrophia ventriculi sin. cordis. Atherosclerosis levis aortae et arteriarum baseos cerebri. Apoplexia sanguinea hemisphaeri dextri cerebelli et infiltratio haemorrhagica leptomeningum, praecipue baseos cerebri. Contusio veterior circumscripta faciei inferior. lobi temporalis sin. Bronchitis. Pleuritis chron. adhaesiva sin. 
Fall 18. S. K, 25jähriges Mädchen, verlor im Sommer 1910 plötzlich das Bewusstsein, hatte, als sie bald wieder zu sich kam, starke Kopfschmerzen und glaubte seitdem eine Abnahme des Gedächtnisses zu bemerken.

Am 19. III. 1911 fiel sie unter lautem Aufschrei um, wurde bewusstlos gefunden und erwachte erst nach mehreren Stunden, blieb aber bis zur Aufnahme in die Klinik bettlägerig.

23. III. Bei der Einlieferung in die Klinik Klagen über Kopfschmerz, auffallende Nackenstarre, Druckempfindlichkeit der ganzen Wirbelsäule. Motorische Reflexe etwas gesteigert.

26. III. Lumbalpunktion ergab stark blutigen Liquor mit leichter Xanthochromie.

In den folgenden Tagen fühlte sich Pat. wohler, klagte nur zuweilen noch über Kopf- oder Rückenschmerzen und hielt auch beim Sitzen oder Stehen den Kopf nach hinten gestreckt.

1. IV. Pat. setzte sich morgens beim Waschen plötzlich auf den Boden und jammerte, sie könne sich nicht aufrecht halten. Sie wurde ins Bett getragen, bot aber keine auffälligen, objektiven Symptome.

3. IV. Lumbalpunktion: Liquor stark braun, enthält keine roten Blutkörperchen.

10. IV. Während der ganzen Beobachtungsdauer stets etwas Nackenstarre, Aufsitzen nur mit angezogenen Knien möglich.

12. IV. Seit gestern plötzlich Doppelsehen. Rechtseitige $A b-$ duzenslähmung. Albernes, läppisches Betragen und sehr launisches Wesen.

An den folgenden Tagen hielt die psychische Veränderung an, ohne dass Pat. dafür einen Grund anzugeben wusste. Zugleich schrie sie manchmal plötzlich laut auf, ebenfalls ohne eine bestimmte Ursache dafür nennen zu können.

15. IV. Mittags plötzlicher heftiger Aufschrei, motorische Unruhe, Greifen nach dem Kopfe, dann tiefes Coma, äusserst blasses Gesicht und völlige Pulslosigkeit. Allmählich eintretendes Würgen und Erbrechen. Nach 2 Stunden Besserung der Herztätigkeit, aber krampfartige Bewegungen der Extremitäten und Zuckungen der Gesichtsmuskeln, deutliche Krämpfe der Kaumuskulatur. Nach weiteren 3 Stunden: Cheyne-Stokesscher Atemtypus. Während des Abends allgemeine Besserung, allmähliches Erwachen, dann Klagen über äusserst heftige Schmerzen im Hinterkopf, starker Opisthotonus. Augenhintergrund normal.

Im Laufe der folgenden Tage trat allmählich zunehmende Benommenheit auf; Pat. griff häufig nach dem Kopf, begann zu fiebern und hatte dauernd eine ziemlich hohe Puls- and Atemfrequenz, Deutliche Nackenstarre.

24. IV. Schnelles Auftreten eines Decubitus am Kreuzbein.

27. IV. Lumbalpunktion: stark bluthaltiger Liquor mit reichlichen roten Blutkörperchen, mehreren Hämatoidin-Kristallen und sehr ausgesprochener Xanthochromie.

2. V. In den letzten Tagen schluckte Pat. nicht mehr. Küustliche Ernährung per os und anum sehr schwierig. Rascher Kräfteverfall und heute Exitus letalis.

Sektion (L.-Nr. 662/1911; Obduzent: Herr Privatdozent Dr. Versé): Schädeldach längsoval, symmetrisch, an der Innenfläche glatt. Dura 
nicht besonders stark gespannt, ihre Gefässe mässig gefüllt. Die Gehirnwindungen sind nicht abgeplattet, die Gefässe der Pia stark gefüllt. An der Basis des Hirnstammes sind die weichen Häute diffus gelblich gefärbt, besonders um den Hypophysenstiel herum. Rechts neben der Arteria basilaris sitzt ein reichlich kleinbohnengrosser, derber Knoten, der fest mit der Dura zusammenhängt. Wie sich bei der Herausnahme des Gehirns zeigt, hat er den Nervus abducens nach abwärts gedrängt und besitzt eine Länge von $1,6 \mathrm{~cm}$ und eine Breite von $1,1 \mathrm{~cm}$. Dieser Kopf hängt unmittelbar mit einer von der Art. basilaris abgehenden Arterie zusammen, welche die Art. cerebellaris ant. darstellt, deren Wand direkt in das im übrigen unregelmässig gestaltete Knötchen übergeht. An der Teilungsstelle dieser Arterie findet sich etwa in der Nähe der Vereinigung beider Art. vertebrales noch eine etwas über stecknadelkopfgrosse, sackartige Ausbuchtung zwischen den beiden Ästen. Auf einem Querschnitt durch den Knopf zeigen sich die äusseren Schichten von gelblichen Thrombusmassen gebildet, die eine Dicke von $3-4 \mathrm{~mm}$ erreichen. Dann gelangt man in einen mit flüssigem Blut gefüllten, kleinerbsengrossen Hohlraum, der unmittelbar mit dem Arterienstamm zusammenhängt. An der Unterfläche der rechten Kleinhirnhemisphäre sind die Venen stark geschlängelt. Die Kleinhirnsubstanz erscheint neben dem Flocculus etwas erweicht. Die Arterien der Basis sind leicht weisslich verdickt. Im Duralsack des Rückenmarks findet sich blutige Flüssigkeit und an der Austrittstelle der Nerven bräunliche Pigmentierung. Der rechte Nervus abducens ist etwas nach hinten und aussen gedrängt und verläuft an der Hinterfläche des kleinen Sackes nach vorn und oben; er ist gewissermassen in die Wand des Sackes eingelassen, verbreitert und fest fixiert. Sein hinterer $\mathrm{Ab}$ schnitt liegt hinter den sich gabelnden Ästen und der Unterfläche des Pons, so dass er nicht gut sichtbar ist.

Sektionsdiagnose: Aneurysma arteriae cerebelli inf. ant. dextr., nervum abducentum dextrum comprimens. Pigmentatio fusca leptomeningum baseos cerebri. Contenta sanguinolenta subarachnoidalia et interduralia medullae spinalis. Decubitus superficialis recens sacralis. Kolpitis. Haemorrhagia mucosae coli.

Während in dem ersten der drei soeben angeführten Fälle (Fall 16) während der klinischen Beobachtung an ein Hirnaneurysma nicht gedacht worden war, konnte in den beiden anderen Fällen das Vorhandensein einer wiederholten meningealen Blutung mit Sicherheit ausgesprochen werden, und im Fall 18 wurde sogar bestimmt die Diagnose auf ein kleines Aneurysma in der hinteren Schädelgrube gestellt. Leider vermochte im Fall 16 auch die Obduktion nicht sicher das Vorhandensein eines Aneurysma nachzuweisen, wie es klinisch ebenfalls vermutet worden war, weil die Blutung allzu weitgehende Zerstörungen angerichtet hatte. Gegen eine einfache Gefässzerreissung sprach aber das wiederholte Auftreten der Blutung und das Fehlen irgendwie erheblicherer Gefässveränderungen, zwei Tatsachen, die sich besser mit der Annahme eines kleinen kongenitalen Aneurysma vereinigen lassen. 
Aus allen drei Krankengeschichten (Fall 16-18) ist jedenfalls als besonders wichtig und charakteristisch hervorzuheben: Das plötzliche Auftreten schwerer, cerebraler Erscheinungen, das Zurückbleiben starker Kopfschmerzen mit ausgesprochener Nackenstarre, die Wiederholung dieser Zustände nach verschiedenen Zeiträumen und endlich der jedesmal typische Befund von zum Teil veränderten roten Blutkörperchen, von sog. Xanthochromie und einer sekundären Lymphocytose (infolge meningealer Reizung) im Liquor cerebrospinalis.

Bei ausreichend kritischer Bewertung solcher Symptome wird zweifellos in Zukunft die Feststellung eines cerebralen Aneurysma intra vitam öfter, als bisher, gelingen. Es soll dabei jedoch nicht geleugnet werden, dass gerade die Lumbalpunktion auch einmal irreführend sein kann, wie z. B. in den von Köster und Thyne berichteten Fällen, weil eben keine meningeale Blutung zustande kam oder das Blut noch nicht bis zur Lendengegend gelangt war. Überhaupt soll nicht verkannt werden, dass trotz scheinbar noch so sicherer Symptome Irrtümer vorkommen können (vgl. u. a. v. Steinmann), und auf diese Möglichkeit wollen wir daher noch etwas näher eingehen.

Grössere Schwierigkeiten können ja vor allem Hirntumoren bedingen, die bekanntlich sowohl wiederholte, apoplektiforme Insulte, als auch Nackenstarre oder sonstige auf ein Aneurysma deutende Erscheinungen hervorzurufen imstande sind. Wir wissen ferner auch, dass bei ihnen Blutungen durch Arrosion von Gefässen zustande kommen können, meist finden diese jedoch in die Substanz der Geschwulst selbst oder in das Gehirnparenchym statt und wohl nur selten in die Meningen. Im letzteren Falle wird meist der Tod die unmittelbare oder doch baldige Folge sein, weil die Aussichten auf eine Thrombose des eröffneten Gefässes nicht sehr gross sind.

Diese Verhältnisse liegen nun bei den Hirnaneurysmen insofern etwas anders, als hier auffällig häufig der Gefässsack schon sehr frühzeitig mit dichten Thrombusmassen angefüllt ist. Die Blutung aus einem solchen Gebilde erfolgt daher meist langsamer und mehr sickernd, und daraus erklärt es sich, dass sie leichter wieder zum Stehen kommt und dass bei Hirnaneurysmen eine mehrfache Ruptur und Blutung etwas nicht ganz Ungewöhnliches ist. Wie schon an anderer Stelle ausgeführt worden ist ${ }^{1}$ ), scheint eine Durchsicht der Literatur zu ergeben, dass nahezu in einem Drittel (31,1 Proz.) aller Fälle von Hirnaneurysmen mindestens eine zweimalige Blutung stattfindet,

1) S. Münch. med. Wochenschr. 1911. Nr. 51. 
und zwar fast stets, wie erwähnt, in die Meningen und nur sehr selten, wie bei den Geschwülsten, in die Hirnsubstanz allein.

Neben solehen, für die Diagnostik wichtigen Merkmalen, die durch Bau und Lage der Hirngeschwülste und -aneurysmen bedingt sind, gibt es noch einen weiteren Unterschied zwischen ibnen, der durch die Grössenverhältnisse und die Wachstumsgeschwindigkeit hervorgerufen wird. Die Aneurysmen der Hirngefässe pflegen nämlich durchweg im Gegensatz zu den Tumoren nur ausnahmsweise einen grösseren Umfang als den einer Erbse oder Bohne zu erlangen, wenn auch solche von recht stattlicher Grösse beobachtet worden sind. Gerade diese ungewöhnlich grossen Hirnaneurysmen scheinen aber wiederum nur sehr langsam heranzuwachsen, weil eben sehr rasch sich vergrössernde Gefässgeschwülste meist auch frühzeitig zum Platzen gelangen und so vor Erreichung eines beträchtlichen Umfangs den Tod des Individuums bedingen. Diesen beiden Umständen ist es zu danken, dass bei den Hirnaneurysmen die Aussichten auf Entwicklung allgemeiner Hirndrucksymptome viel geringer sind, als bei cerebralen Geschwïlsten, und das Auftreten solcher Erscheinungen spricht daher mehr für das Vorhandensein letzterer (vgl. Uhthoff).

In der älteren Literatur unseres Gegenstandes begegnen wir häufig der Angabe, dass Hirnaneurysmen durch das Auftreten eines subjektiv und objektiv hörbaren Geräusches am Kopfe gekennzeichnet seien. Es gibt eine grössere Zahl von Veröffentlichungen, in denen sich die Diagnose einer solchen Gefässgeschwulst fast allein auf dieses Symptom stützte. Unter ihnen seien die Arbeiten von Cö̈, Humble, Hutchinson, Maclaren, Mecrkowski, Möser (Fall 2), v. zur Mühlen, Puzey und Schalkhauser genannt, ron denen jedoch nur die beiden Fälle von Hutchinson und Möser später durch die Obduktion als Hirnaneurysmen sichergestellt worden sind. Wir wissen nämlich heutzutage, dass es sich beim Auftreten solcher Geräusche um eine recht vieldeutige Erscheinung handelt, die bei Hirntumoren, Morbus Basedowii, schwerer Anämie, ja, bei Kindern sogar normalerweise auftreten kann. Für die Diagnostik der Hirnaneurysmen dürfen wir daher solchen Geräuschen nur noch sehr beschränkten Wert zumessen, wie es auch Beadles in seiner ausführlichen Arbeit feststellen konnte.

Aus alledem ergibt sich also, dass zwar einige klinische Erscheinungen, unter denen mehrfache meningeale Blutungen besonders hervorzuheben sind, mit grosser Wahrscheinlichkeit auf das Vorhandensein eines Hirnaneurysma hindeuten, andere Symptome aber doch zu vieldeutig sind, um diese Annahme zu stützen, ja, einige sogar, wie z. B. ausgesprochene Hirndruckerscheinungen, geradezu irreführend 
sein können, indem sie bei Tumoren weit häufiger sind, als bei Aneurysmen. Wir werden daher zugeben müssen, dass bisher kein völlig sicheres Symptom für Hirnaneurysmen bekannt ist, und dass die Annahme eines solchen fast immer nur eine Wahrscheinlichkeitsdiagnose sein wird.

Durch diese Erkenntnis werden wir uns nicht abhalten lassen, im Einzelfalle tatsächlich mit einiger Bestimmtheit diese Diagnose zu stellen, und es erwächst uns dann daraus die weitere Aufgabe, den genaueren Sitz des Aneurysma festzustellen. Dabei treten uns allerdings neue und nicht unerhebliche Schwierigkeiten entgegen. Es soll nicht geleugnet werden, dass gerade in der letzten Zeit bedeutende Fortschritte in der Lokalisation cerebraler Affektionen erzielt worden sind, und es darf andererseits auch nicht übersehen werden, dass die Hirnaneurysmen trotz ihrer Kleinheit durch ihr besonders häufiges Vorkommen an der Hirnbasis verhältnismässig oft ziemlich bestimmte Ausfallserscheinungen, namentlich durch Druck auf einzelne Hirnnerven, hervorrufen. Trotzdem werden aber die Symptome selten so einwandfrei sein, dass daraufhin z. B. ein operativer Eingriff gewagt werden könnte.

Es ist nämlich allein schon daran zu denken, dass die von einem Hirnaneurysma abgehenden Gefässäste häufig thrombosieren. Versorgt nun ein solcher Ast wichtige Gehirnbezirke, so ruft ihr Ausfall infolge unzureichender Ernährung schwere klinische Erscheinungen hervor, die also gar nicht unmittelbar durch die Gefässgeschwulst selbst entstehen, sondern gewissermassen als eine "Fernwirkung“ dieser aufzufassen sind. Es ist unter solchen Umständen leicht begreiflich, dass schwere lokalisatorische Irrtümer möglich sind.

Weiterhin dürfte noch kurz die Möglichkeit in Erwägung zu ziehen sein, dass die von einer früheren Blutung aus dem Aneurysma stammenden Gerinnsel durch Druck, Schrumpfung u. dgl. einmal Reiz- oder Ausfallssymptome bedingen könnten; demgegenüber muss aber nach einer daraufhin gerichteten Durchsicht der Literatur betont werden, dass wohl nur bei ganz profusen und daher fast immer schnell tödlichen Blutungen grössere Gerinnselbildungen im Liquor cerebrospinalis (wenigstens an der Gehirnbasis) vorkommen und dass sich bei Aneurysmen kleinere Coagula meist nur in unmittelbarer Nachbarschaft der Gefässgeschwulst selbst bilden und dann eher den Sitz dieser verraten, als eine Täuschung hervorrufen können. Es ist ja kein seltenes Ereignis, dass ein perforiertes Hirnaneurysma durch Anlagerung thrombotischer Massen vergrössert wird und nunmehr einen Druck auf einen benachbarten Hirnnerven ausiiben kann. Fall 18, bei dem einige Zeit nach einer sicheren Blutung eine Lähmung des 
rechten Nervus abducens auftrat, kann viclleicht als Beispiel hierfür gelten.

Immerhin wird nach den vorhergehenden Betrachtungen die Feststellung des genaueren Sitzes eines Hirnaneurysma fast stets grosse Schwierigkeiten haben, und es wird anch bei anscheinend sehr eindeutigen Symptomen Grund genug zur Vorsicht vorliegen. Diese ist um so mehr am Platze, als die in der Literatur niedergelegten Erfahrungen zeigen, dass nicht ganz selten zwei oder mehrere Hirnaneurysmen bei einem Kranken bestehen, und dass andererseits neben einem tatsächlich vorhandenen Aneurysma auch meningeale Blutungen aus einem nicht erweiterten Gefässe vorkommen können. Dadurch ist eine ausserordentlich grosse Fülle von Möglichkeiten gegeben, die zu praktisch recht bedenklichen Täuschungen führen können und im einzelnen hier wohl nicht näher erörtert zu werden brauchen. Nur ein recht anschauliches Beispiel dieser Art sei durch Mitteilung folgender Beobachtung kurz hier angeführt:

Fall 19. A. L., 56 jährige Witwe, zog sich vor 8 Jahren angeblich infolge einer Verletzung eine Entzündung und später ein Geschwür am rechten Unterschenkel zu, das nur sehr langsam heilte. Vor 3 Jahren bekam sie plötzlich mehrere Tage lang Erbrechen, das von der Nahrungsaufnahme unabhängig war, und litt in dieser Zeit an Schwindelanfällen. Im folgenden Jahre wiederholte sich derselbe Zustand und hielt wieder etwa eine Woche an und ebenso etwa $3 / 4$ Jahre später. Bei diesem dritten Anfalle trat eine Lähmung des linken oberen Augenlides auf, die bis zur $1 / 2$ Jahr später erfolgenden Aufnahme in die Klinik bestehen blieb.

Bei der kräftigen, gut genährten Kranken fand sich zu dieser Zeit ein hirsekorngrosses, zackiges Geschwür des Zungenrückens mit grüngelblichem Belag und am rechten Unterschenkel mehrere etwa talergrosse, pigmentierte Hautnarben. Daneben konnte eine vollständige, linksseitige Ophthalmoplegia externa und interna festgestellt werden, und die Art. temporales erschienen etwas verdickt und leicht geschlängelt. Nach einer fast achtwöchentlichen Schmierkur und Behandlung mit Jodkali verschwanden die linkseitigen Augenmuskellähmungen fast vollständig; auch das Allgemeinbefinden besserte sich, und das frühere, zeitweilig noch vorhandene Erbrechen kehrte innerhalb dieser Zeit nicht wieder. Kurz nach Beendigung der Kur aber stellte sich plötzlich von neuem Schwindelgefühl, Brechreiz und dazu rechtseitige Ptosis und Facialislähmung ein; jedoch gingen diese Erscheinungen in $21 / 2$ Wochen nach weiterer Verabreichung von Jod wieder zurück. Acht Tage später wurde Pat. unter den Zeichen eines apoplektischen Insultes plötzlich rechtseitig gelähmt. Der weitere Verlauf bot keine bemerkenswerten Erscheinungen; es trat eine Kontraktion des rechten Armes und Beines ein, wảhrend sich die Kranke im übrigen leidlich erholte. Funf Monate nach dem zuletzt erwähnten Schlaganfalle kam Pat. unter erneuten apoplektiformen Symptomen plötzlich ad exitum. 
Sektion (L.-Nr. 742/07; Sektionskurs):

Nach Entfernung des Schädeldaches zeigt sich das Gehirn mächtig ausgedehnt; die weichen Häute sind an der Konvexität verdickt, und entlang der beiden Fossae Sylvii verbreitet sich in ihnen frisch ergossenes Blut. Bei der Herausnahme des Gehirns zeigt sich an der Basis reichlich frisches, dunkles Blut, besonders in der Gegend des Chiasma opticum. Die Art. carotis interna, deren Durchschnitt etwas klafft, ist, ebenso wie alle anderen Hirnarterien, etwas dicker und weisser als gewöhnlich. An der Abgangsstelle der Art. cerebri post. von der Art. basilaris findet sich eine etwa $8 \mathrm{~mm}$ weite, sackförmige Ausbuchtung der Arterienwand; im vorderen Teile ist ihre Wand etwas dünner und zeigt hier ein von oben nach unten verlaufendes, etwa $3 \mathrm{~mm}$ langes Loch. An der linken Seite des Sackes befindet sich dicht vor ihm und dem linken Nervus oculomotorius ein derbes, grauweisses Knötchen, das mit der Gefässwand zusammenhängt und in das eine kleine Ausbuchtung des Sackes mündet. Vom rechten Umfange des Sackes verzweigen sich reichliche, kleine und auffallend dicke Âstchen und $11 \mathrm{~mm}$ hinter ihm liegt an der linken Seite der Art. basilaris noch ein zweiter, kleiner, aber leerer Sack von etwa $3 \mathrm{~mm}$ Durchmesser. An der Hauptteilungsstelle der linken Art. fossae Sylvii in ihre Äste ist die ganze Arterienwand sehr dick und weisslich; das Rohr selbst ist stark verlegt, aber nicht ganz verschlossen. Auch die Äste der rechten Art. fossae Sylvii zeigen mehrfache, ähnliche Verdickungen, und einzelne sind beiderseits auch an der Art. cerebri ant. und corporis callosi zu finden. Die Seitenventrikel des Gehirns sind erweitert und mit blutig gefärbter, wässeriger Flüssigkeit gefüllt; ihr Ependym ist etwas granuliert. In der Mitte des linken Streifenhügels findet sich ein grösserer, etwas eingesunkener, gelblicher Herd. Der dritte und vierte Ventrikel enthalten ebenfalls blutige Flüssigkeit, und im Foramen Magendii findet sich ein dunkelrotes Blutgerinnsel. Der Duralsack des Rückenmarks enthält nur mässige Mengen Blutes.

Sektionsdiagnose: Syphilis obsoleta. Cicatrices cutis cruris sin. Hyperostosis tibiae sin. Endarteriitis syphilitica arteriar. cerebri. Aneurysma recens perforatum arteriae basilaris cerebri (ad ostium art. cerebri post. sin.). Arteriosclerosis partim fibrosa arcus aortae. Encephalomalacia veterior flava circumscripta lobi temporal. sin. et gangliorum hemisphaerii sin. cerebri. Emphysema pulmon. et induratio circumscripta nigra apicis pulm. dextri utriusque. Fibroma. ovar. dextri. Arthritis deformans incipiens genu dextri. Cholelithiasis. Cicatrices vesicae felleae.

Eine diffus, auf alter Syphilis beruhende Arterienerkrankung des Gehirns hatte hier zur Bildung ron zwei von einander getrennter Aneurysmen der Arteria basilaris geführt, von denen eines perforiert war und dadurch den Tod der Kranken bedingte. Es ist möglich, vielleicht sogar wahrscheinlich, dass die in der Anamnese angegebenen, früheren Anfälle von Schwindelgefühl und Erbrechen auf leichten und sickernden Blutungen aus diesem letzteren Aneurysma beruhten. Jedenfalls lassen sich aber die linkseitigen Augenmuskelstörungen auf unmittelbare Nervenkompression durch dasselbe Aneurysma zurück- 
führen. Wenn diese Erscheinungen nach antisyphilitischer Behandlung zurückgingen, so beruht das wohl auf einer Verkleinerung des Umfangs der Gefässerweiterung; dem entspricht es ja, dass bei der Sektion eine kleine Ausbuchtung des Aneurysma gefunden wurde, die mit dem N. oculomotorius zusammenhing und eine besonders derbe Wand zeigte. Interessant ist es nun, dass ganz unabhängig von den Gefüssgeschwülsten ausserdem noch eine Blutung durch einfache Gefässzerreissung in dem linken Streifenhügel stattgefunden hatte. Durch ein solches Ereignis kann sehr leicht eine Täuschung über den Sitz eines sonst richtig diagnostizierten Aneurysma hervorgerufen werden.

Diese Möglichkeit ist vor allem dann gegeben, wenn, wie in diesem Falle, eine schwere und ausgedehnte Gefässerkrankung des Gehirns vorliegt. Andererseits darf man aber auch bei jugendlichen Personen, bei denen ein solches allgemeines Befallensein der Hirnarterien fast ausgeschlossen erscheint, nicht vergessen, dass, wie Eppinger schon gezeigt hat, auch auf kongenitaler Anlage entstandene Aneurysmen nicht selten zu mehreren auftreten und dass dann das eine von ihnen durch Druck, ein anderes durch Blutung, ein drittes durch Gefässverlegung Erscheinungen machen kann. Um nur einige hierher gehörige, interessante Beobachtungen aus der Literatur zu nennen, sei auf die Veröffentlichungen von Gull (Fall 5), G. Marchant, Nothnagel, Ord, Russel verwiesen.

Vergegenwärtigen wir uns alle diese Hindernisse, die einer sicheren und genauen Lokalisation eines Hirnaneurysma entgegenstehen, so werden wir es verstehen, dass sich, wie schon in der Einleitung kurz berührt wurde, die Chirurgie der Therapie dieses Leidens bisher noch wenig angenommen hat. Bei den Versuchen, die Aneurysmen der Carotis interna durch Unterbindung der Carotis communis zu heilen, was in einigen Fällen mit Erfolg gelang, fehlte es leider meist an einer späteren Bestätigung der Diagnose durch die Sektion, und diese Fälle lassen sich daher nur mit Vorsicht verwerten. Da zudem die Unterbindung der Carotis communis doch einen recht gewagten Eingriff darstellt, scheint man neuerdings von dieser Art der Therapie wieder abgekommen zu sein. Jedenfalls gehören die Mitteilungen darüber vorwiegend der älteren Literatur an.

Über therapeutische Erwägungen ist im übrigen, abgesehen von der Erörterung solcher chirurgischer Versuche, kaum etwas zu finden. Und doch sollte man denken, dass, wenn man die Diagnose auf ein Hirnaneurysma gestellt hat, von systematisch fortgesetzten subkutanen Gelatineinjektionen, die sich offenbar doch bei manchen Aortenaneurysmen bewährt haben, ein gewisser Erfolg erzielt werden könnte. Wir wissen, dass dadurch die Gerinnungsvorgänge des Blutes geför- 
dert werden, und gerade die Hirnaneurysmen neigen ja sehr stark zur Thrombosierung. Dass beim Auftreten von Blutungen aus der Gefässgeschwulst, die sich durch die früher erwähnten Anzeichen verraten, grösste körperliche Ruhe und Vermeidung aller den Blutdruck steigernden Einflüsse notwendig ist, braucht nicht näher erörtert zu werden. Im allgemeinen müssen wir aber bekennen, dass wir diesem Leiden ziemlich machtlos gegenüberstehen.

Wenn es uns demnach auch an einem wirksamen Mittel oder Verfahren zur Beseitigung der Hirnaneurysmen fehlt, so dürfen wir doch nicht ausser acht lassen, dass schon dadurch etwas gewonnen sein würde, dass wir nach richtiger Diagnosenstellung schädliche Massnahmen und Einflüsse vermeiden. Da schwerere cerebrale Erscheinungen nicht selten sind, so liegt die Gefahr einer Verwechslung mit Vergiftungen, urämischen Zuständen, Coma diabeticum usw. sehr nahe, und wir könnten uns veranlasst sehen, in solchen. Fällen, blutdrucksteigernde Kocbsalzinfusionen, künstliche. Atemübungen, Darreichung starker Herzmittel und ähnliches vorzunehmen, wodurch eine Blutung aus dem Aneurysma nur begünstigt wurde. Es wird sich deshalb empfehlen, bei Kranken mit schweren Hirnerscheinungen stets auch an die Möglichkeit eines cerebralen Aneurysma zu denken und daraufhin zu untersuchen.

Diese Mahnung erscheint einmal deshalb besonders berechtigt, weil in den meisten veröffentlichten Fällen zugestandenermassen dieses Leiden gar nicht in den Bereich der klinischen Betrachtungen gezogen worden war, ausserdem aber gibt es eine grössere Zahl ron Mitteilungen, die zeigen, dass zufällige Begleitumstände den Untersucher auf Irrwege leiteten und zu einer ganz falschen Annahme gelangen liessen. Auch in dieser Beziehung konnten in unserer Klinik einige interessante Erfahrungen gesammelt werden, und im folgenden soll daher noch die Mitteilung von drei weiteren Krankengeschichten erfolgen, die uns solche diagnostische Irrtümer begreiflich zu machen geeignet sind:

Fall 20. E. N., 51jährige Punktiererin, ist bisher im allgemeinen gesund gewesen, hatte aber in der letzten Zeit bei Anstrengungen Kurzatmigkeit. Am Morgen des 19. XI. 1908 brach sie auf dem Wege zur Arbeit bewusstlos zusammen und wurde so in die Klinik geschafft. Es handelte sich um eine gut genährte, kräftige Frau, die in tiefstem Coma lag. Sie bekam anfallsweise allgemeine tonische und klonische Zuckungen, dazwischen klonische Krämpfe der Gesichtsmuskulatur allein. Die Pupillen waren eng und reagierten kaum sichtbar auf Lichteinfall. Am Herzen fand sich eine leichte Dilatation und ein etwas hebender Spitzenstoss. Die Atmung war stertorös, der Puls zeigte 110 Schläge, Fieber bestand nicht. Im Urin fanden sich $1 \%$ Eiweiss und spärliche granulierte Zylinder, 
sowie einige Leukocyten. Das Sensorium hellte sich nach einem Aderlass auf; abends trat aber wieder für kurze Zeit plötzlich Bewusstlosigkeit mit allgemeinen klonischen Zuckungen auf. Am folgenden Morgen nach gut verbrachter Nacht trat plötzlich wieder Bewusstlosigkeit mit stark vertiefter Atmung und 3 Minuten später der Tod ein.

Sektion (L.-Nr. 1588/08; Obduzent: Herr Dr. Knierim):

Schädeldach längsoval, symmetrisch, von glatter Innenfläche. Die Dura ist ziemlich gleichmässig prall über beiden Hemisphären gespannt. Die Pia ist an der Konvexität ein wenig getrübt, in der Gegend der Fossa Sylvii und an der ganzen Gehirnbasis mit reichlichen Cruormassen durchsetzt, die besonders dem Verlaufe der Gefässe folgen. In den Fossae Sylvii und in der Gegend der Tractus optici sind die Cruormassen besonders stark angehäuft. Die Arteria basilaris ist nahe der Teilungsstelle verdickt und fühlt sich fest an; auf dem Durchschnitt zeigt sich das Lumen nur von sehr geringer Weite. Die Intima ist glasig-grau durchscheinend und sehr erheblich verdickt. Die gleichen Veränderungen zeigen die Art. cerebri anteriores, und an der linken Art. cerebri anter. ist nahe der Ursprungsstelle eine kleinerbsengrosse, sehr dünnwandige Ausstülpung der Arterienwand vorhanden. In diesem ausgebuchteten Säckchen findet sich eine stecknadelkopfgrosse öffnung, die nach vorn und unten zu liegt. Die kleinen Hirnarterien zeigen an einigen Stellen gelbe, sklerotische Plaques in ihrer Wandung.

Sektionsdiagnose: Endarterititis obliterans syphilitica arter. basilaris. Aneurysma perforatum arteriae cerebriant. sin. Haemorrhagiae diffussae baseos cerebri et fossae Sylvii. Defectus mammae sin. et cicatrix cutis ibidem. Tuberculosis obsoleta lobi sup. pulm utriusque. Struma colloides.

Fall 21. M. U., 52jähriger Monteur, der bisher gesund gewesen war, fiel auf einem Geschäftsgange plötzlich um, wurde in ein Haus geschafft und äusserte, als er nach 10 Minuten zum Bewusstsein kam, den Wunsch, in seine Wohnung gebracht zu werden. Bald darauf versank er wieder in Bewusstlosigkeit und kam in diesem Zustande in die Klinik. Eine genauere Nachforschung ergab, dass Pat. schon längere Zeit öfter an "Migräne" mit Flimmern vor den Augen und Erbrechen gelitten hatte. Bei der Einlieferung am 20. I. 05 wurde Somnolenz bei erhaltenem Sprachvermögen und Fehlen von Lähmungen festgestellt. Pat. liess sehr bald reichlich Urin, der $1 / 2 \%$ Zucker und eine nicht messbare kleine Menge Eiweiss enthielt; im Sediment fanden sich aber einige hyaline, granulierte und epitheliale Zylinder, sowie einzelne weisse und rote Blutkörperchen. Pat. klagte über heftige Kopfschmerzen und erbrach mehrere Male. Der Puls zeigte $60-78$ Schläge, die Temperatur war normal.

An den beiden folgenden Tagen war noch etwas Zucker im Urin nachweisbar, später nicht mehr; dagegen hatte Pat. dauernd Spuren Eiweiss bei einer Menge des Harns von etwa 1 Liter in 24 Stunden und einem spezifischen Gewichte von 1021. Die Allgemeinerscheinungen, besonders die Kopfschmerzen, besserten sich täglich; der Augenhintergrund war normal. Am 31. I., also 11 Tage nach der Aufnahme, fing Pat. plötzlich an zu röcheln, warf bräunlich-schaumige Flüssigkeit aus und wurde dabei für kurze Zeit bewusstlos. Der ganze Anfall dauerte 10 Minuten, und 
hinterher hatte Pat. starke Kopfschmerzen. Nach etwa 6 Stunden trat ein ganz ähnlicher Anfall auf; der schaumige Auswurf war reichlicher, Pat. blieb aber, als diese Erscheinung nach einigen Minuten wieder aufhörte, bewusstlos und starb innerhalb einer halben Stunde.

Sektion (L.-Nr. 124/05; Obduzent: Herr Dr. Risel):

Schädeldach lang und schmal, frei von äusseren Verletzungen. Beim Aufsägen des Schädels wird der Duralsack rechts etwas angeschnitten, und dabei entleert sich dunkles, flussiges Blut. Das Gehirn wird durch einen Horizontalschnitt in Höhe des Sägeschnitts zerlegt. In den weichen Häuten der rechten Hemisphäre findet sich reichlich meist noch flüssiges Blut. Beide Seitenventrikel sind weit und frei; ebenso zeigen die grossen Ganglien keine Veränderungen. Im rechten Schläfenlappen ist eine etwa $4-5 \mathrm{~cm}$ lange, schräg von der Spitze des Schläfenlappens bis gegen das Hinterhorn hinreichende Höhle getroffen, die von frischem Coagulum erfüllt ist, deren Wand aber von sehr wenig verändertem Hirngewebe gebildet wird. Nur nach vorne zu ist die Konsistenz der Gehirnsubstanz sehr weich und das Aussehen mehr hämorrhagisch. Besonders reichlich ist die blutige Infiltration der weichen Häute in der Gegend der Fossa Sylvii. Bei der Herausnahme der unteren Hälfte des Gehirns findet sich in der vorderen und mittleren rechten SchädeJgrube reichlich frisches, dunkles Coagulum. Um den Stamm der rechten Art. carotis int. und der rechten Art. fossae Sylvii sind die Coagula etwas fester und mehr bräunlich. Etwa dicht an der Teilungsstelle der rechten Art. fossae Sylvii findet sich an dem Hauptstamme nach unten und hinten zu eine etwa $5 \mathrm{~mm}$ im Durchmesser haltende Ausbuchtung der Wand, in deren Umgebung die Coagula besonders fest haften und auch die Gehirnsubstanz sehr weich und stark durchblutet ist. Von dem Hauptstamme der Art. fossae Sylvii gelingt es ohne Schwierigkeit mit der Sonde in diesen Sack und von da aus in die Höhle an der Spitze des rechten Schläfenlappens vorzudringen. Bei genauerem Zusehen zeigen sich die Häute in der Umgebung des Sackes sehr stark von Blut infiltriert; die Wand des Sackes selbst ist sehr dünn und an einer kleinen Stelle auf der Höhe durchrissen. An der diesem Riss entsprechenden Stelle ist die Gehirnsubstanz vollständig zerstört. An der unteren Hälfte des Gehirns findet sich in der Nachbarschaft der Höhle ein walnussgrosses, bräunliches Gerinnsel. Die Arterien an der Basis sind zart und dünnwandig und nur an einigen Stellen mit kleinen, fleckigen Einlagerungen versehen.

Sektionsdiagnose: Arteriosklerosis levis aortae. Aneurysma sacciforme art. fossae Sylvii dextrae recens perforatum. Haematoma extrameningeale baseos cerebri. Haemorrhagia lobi temporalis hemisphaeri dextri cerebri et infiltratio haemorrhagica piae matris hemisphaeri dextri cerebri. Hyperaemia palmonum. Tuberculosis absoleta et calcificatio glandul. lymphaticar. mesenterii. Foramen ovale apertum. Phlebolithi venarum plexus prostatici.

Fall 22. F. Sch., 38jährige Ehefrau, litt seit dem 19. Lebensjahre an Basedowscher Krankheit. Am Tage der Einlieferung (2. März) wurde sie mittags bewusstlos im Zimmer gefunden, das dicht mit Rauch aus einem leergebrannten Petroleumbrenner erfüllt war. Die kräftige Frau zeigte cyanotische Gesichtsfarbe, dyspnoiscne Atmung und tiefstes Coma. 
Am Herzen war eine deutliche Dilatation nach links und eine sehr hohe Pulsfrequenz festzustellen, daneben bestand starker Exophthalmus und eine deutliche Struma. Im Urin fand sich etwas Zucker. Nach sofortigem Aderlass und Sauerstoffinhalation besserte sich der Allgemeinzustand, und abends schien Pat. ihren Mann schon zu erkennen. Eine kurz nach der Aufnahme in der Klinik entommene Blutprobe zeigte spektroskopisch normales Verhalten. Die Kranke war im Laufe dieses Tages sehr unruhig, warf sich viel umber, und erst am folgenden Morgen wurde sie ruhiger. Es zeigte 'sich dann, dass sie deutliche, wenn auch nicht sehr erhebliche Nackenstarre hatte und bei Druck auf die Halswirbelsäule schmerzhaft das Gesicht verzog. Der Augenhintergrund war normal; eine Lumbalpunktion ergab ein "negatives Resultat“.

Allmählich verschwand die Nackenstarre, Pat. erkannte nach zwei weiteren Tagen ihre Angehörigen, blieb aber leicht benommen. 5 Tage nach der Aufnahme trat Fieber auf, und die motorische Unruhe, die dauernd geblieben war, wurde stärker. Am nächsten Tage trat plötzlich tiefe Bewusstlosigkeit, und bald darauf der Tod ein.

Sektion (L.-Nr. 264/06; Obduzent: Herr Dr. Risel):

Schädeldach ziemlich lang und schmal, seine Nähte verstrichen. Die Innenfläche der Dura ist glatt und glänzend. Im Sinus longitudinalis befinden sich nur Leichengerinnsel, im Sinus tentorii, im Confluens sinuum und im linken Sinus transversus liegt eine ziemlich frische, graurötliche, stellenweise etwas festsitzende Thrombusmasse. Am seitlichen Umfange beider Grosshirnhemisphären verbreiten sich von der Fossa Sylvii aus nach vorn und oben hin grössere oder kleinere, verwachsene Blutaustritte in den weichen Häuten. Sehr viel reichlicher zeigen sich diese bei der Herausnahme des Gehirns um das Chiasma herum und an den beiden Fossae Sylvii. Im vorderen Umfange der Art. communicans ant. findet sich ein $2^{1 / 2}-3 \mathrm{~mm}$ im Durchmesser betragender, knopfförmig hervorragender Sack, an dessen rechtem unteren Umfange ein kleines Loch zum Vorschein kommt, aus welchem bei Druck Blut heraustritt. Die Hirnarterien an der Basis sind überall dünnwandig und zart und zeigen keine Veränderungen. Die Hirnsubstanz ist am vorderen Umfange des Balkens ziemlich weich und leicht zerreisslich. Beide Seitenventrikel, sowie der dritte Ventrikel enthalten schwach blutig gefärbte Flüssigkeit, keine grösseren Gerinnsel, nur im vierten Ventrikel findet sich ein kleines, dunkelrotes Gerinnsel. Die blutige Infiltration setzt sich beiderseits auf die Opticusscheide bis zum Bulbus in Form einer diffusen Rötung fort; bei Aufschneiden der Sehnervenscheide finden sich einzelne kleine, verwaschene Blutansammlungen.

Sektionsdiagnose: Struma parenchymatosa. Protrusio bulbi utriusque. Hypertrophia cordis totius praecipue ventriculi sin. Ruptura aneurysmatis sacciformis art. communicantis anterioris cerebri. Haemorrhagia intrameningealis lateralis. Cysticerci cellulosae obsoleti pulm. utriusque. Atrophia granularis levis renum. Hepatitis chron. interstitialis incip.

Diese drei Beobachtungen sind dadurch ausgezeichnet, dass besondere Umstände bei der Stellung der Diagnose zu einem Irrtum verleiten mussten. Im Fall 20 wurden anfallsweise auftretende Krämpfe 
des ganzen Körpers festgestellt; der Harn wies Eiweiss und Zylinder auf, und nach einem Aderlass trat sichtbare Besserung des Allgemeinbefindens auf. Alle diese Tatsachen mussten bei der kurzen Beobachtungsdauer zu der klinisch am meisten wahrscheinlichen Diagnose einer Urämie führen, die damals auch tatsächlich gestellt wurde.

Nicht viel anders lagen die Verhältnisse im Fall 21, bei dem in den ersten Stunden der Beobachtung wegen des Befundes von Zucker im Urin auch an Coma diabeticum gedacht werden konnte, wenn diese Annahme auch von vornherein nicht sehr wahrscheinlich iwar. Für Urämie sprach dagegen der allmähliche Beginn mit Kopfschmerzen („Migräne") und Erbrechen, die plötzlich mit Bewusstlosigkeit einsetzende Steigerung dieser Erscheinungen und das dauernde Vorhandensein ron Eiweiss und Zylindern im Harn, das vielleicht nicht einmal durch das Ergebnis der Obduktion genügend erklärt worden $\mathrm{zu}$ sein scheint.

Ein zufälliges Zusammentreffen von Umständen, das aber recht wohl begreiflich ist, lenkte schliesslich auch im Falle 22 die Untersuchung in ganz falsche Bahnen. Die 39 jährige Frau mit ausgesprochener Basedowscher Krankheit hatte offenbar den Petroleumbrenner angezündet und war dabei bewusstlos geworden. Als sie aufgefunden wurde, war das Zimmer so dicht mit Qualm und Rauch gefüllt, dass unbedingt eine Rauch- oder Kohlenoxydvergiftung angenommen werden musste und Pat. auch unter dieser Diagnose in die Klinik eingeliefert wurde. Der fehlende Nachweis von Kohlenoxyd-Hämoglobin im Blut sprach ja nicht ohne weiteres dagegen; nur die am folgenden Tage deutlich ausgeprägte Nackenstarre hätte sofort den Verdacht auf ein Hirnaneurysma mit meningealer Blutung wachrufen müssen, abgesehen daron, dass der weitere Verlauf micht mehr mit der Annahme einer Rauchvergiftung in Einklang zu bringen war.

In allen drei Fällen hätte die Lumbalpunktion wohl einen sehr wichtigen differentialdiagnostischen Anhaltspunkt geben können, weil bei ihnen allen wahrscheinlich sofort ergiebigere Blutungen in die Meningen stattgefunden hatten. Während im Falle 20 und 21 überhaupt keine Lumbalpunktion vorgenommen wurde, ergab diese im Falle 22, wie die Krankengeschichte besagt, ein „negatives Resultat". Es ist dabei wohl fraglich, ob nicht tatsächlich blutiger liquor durch die Kanüle entleert wurde und dieser Befund, wie es auch andere Autoren in ihren Fällen nachträglich zugaben, nur fälschlich als Misslingen des Eingriffs gedeutet wurde. -

Nachdem wir so die wichtigsten diagnostischen und therapeutischen Gesichtspunkte unseres Themas unter Herbeiziehung eigener 
Beobachtungen erörtert haben, dürfte es sich empfehlen, am Schlusse der Arbeit eine kurze Übersicht über die hier veröffentlichten Fälle zu geben. Von den 22 mit Hirnaneurysmen behafteten Personen waren 13 Frauen und 9 Männer; diese Verteilung auf beide Geschlechter stimmt mit der Angabe v on Hofmanns überein, dass Frauen häufiger als Männer betroffen sind, wenn man allerdings auch heutzutage nicht mehr zuzugeben vermag, dass dieser Unterschied so beträchtlich ist, wie v. Hofmann es nach seinem Material behaupten konnte. . Ferner starben von jenen 22 Individuen 3 im Alter zwischen 20 und 29 Jahren, je 6 im 4., 5. und 6. Dezennium des Lebens, endlich 1 wurde über 60 Jahre alt. Das entspricht durchaus den Feststellungen anderer Autoren; doch sollte man dabei stets berücksichtigen, dass mit solchen Angaben noch nichts Sicheres über die Zeit, in der das Aneurysma entstanden ist, gesagt ist.

Um darüber einige Klarheit zu gewinnen, müssen wir in jedem Falle nach der Ätiologie des Aneurysma fragen, und selbst dann wird es oft genug zweifelhaft bleiben, eine wie lange Entwicklungsdaver die Gefässgeschwulst gehabt hat. In unseren Fällen waren 5 Aneurysmen sicher auf kongenitaler Anlage entstanden (Fall 5, 6, $7,15,15)$; in 6 weiteren Fällen $(3,4,13,16,21,22)$ kam die gleiche Entstehungsweise jedenfalls sehr wesentlich in Frage, so dass also etwa in der Hälfte unserer Fälle die Aneurysmen schon seit frühester Jugend bestanden haben.

Auf atherosklerotischer Grundlage beruhten dagegen wohl die Gefässerweiterungen im Fall 1 und 17, auf syphilitischer Arterienerkrankung in Fall 19 und 20, und embolischer Natur waren endlich die Aneurysmen der Fälle 8-11, vielleicht auch des Falles 14. Alle diese haben vermutlich eine höchstens mehrjährige Entwicklungszeit durchgemacht, bis sie zum Tode durch Platzen führten.

In sämtlichen Fällen kam es zur Ruptur des Aneurysma; von Interesse ist es aber, dass wohl in 15 von ihnen eine zwei- oder mehrmalige Ruptur mit ganz verschiedenen Zeitabständen von Tagen bis zu Jahren oder Jahrzehnten stattfand. Auf die Bedeutung dieser Tatsache ist ja im Vorstehenden genügend hingewiesen worden, und es sei hier betont, dass gemäss unserer früheren Behauptung sämtliche 22 Aneurysmen mit einer einzigen Ausnahme (Fall 9) meningeale Blutungen verursachten. Im Fall 12 erscheinen allerdings die ersten Perforationen nur zu Hämorrhagien in die Hirnsubstanz geführt zu haben.

Betrachten wir ferner kurz die bei unseren Beobachtungen befallenen Arterien, so ergibt sich, dass die Art. cerebri media am häufigsten, nämlich 8 mal und zwar zu gleichen Teilen rechts und links, 
Sitz der Gefässerweiterung war. Im übrigen war die Art. carotis int. sin. und die Art. cerebelli post. inf. sin. je 2 mal betroffen, alle anderen Arterien, d. h. also die Art. communicans ant. und post., die Art. cerebri ant. dextra und sin., die Art. profunda cerebri dextra, die Art. basilaris, die Art. vertebralis dextra und sin., endlich die Art. cerebelli ant. inf. dextra je 1 mal. Nach einer Zusammenstellung von Hey, die 322 Fälle umfasst, ist neben der Art. cerebri media die Art. basilaris im allgemeinen am häufigsten ausgebuchtet, während z. B. die Kleinhirnarterien nur sehr selten Aneurysmen zeigen. Die Fälle 4, 14, 17, 18 verdienen daher besondere Beachtung.

Werfen wir endlich noch einen Blick auf die Grösse der einzelnen Aneurysmen in unseren Fällen, so finden wir unsere frühere Behauptung bestätigt, dass nur wenige, etwa 4, den Umfang einer Erbse oder Bohne übertrafen. Im allgemeinen gehörten die grösseren Aneurysmen auch den Arterien mit weiterem Durchmesser an. Zu beachten ist aber bei solchen Feststellungen stets, dass manche Hirnaneurysmen von aussen durch angelagerte Thrombusmassen, die aus früheren Blutungen stammen, erheblich grösser erscheinen, als die wirkliche Ausdehnung der Gefässwand an dieser Stelle ist.

\section{Literatur.}

1) Beadles, Brain 1907. Bd. 30. S. 285.

2) Beykovsky, Wiener med. Wochenschr. 1904. S. 825.

3) Cö̈, Gaz. des hôpitaux 1856. S. 495.

4) Eppinger, Arch. f. klin. Chir. 1887. Bd. 35.

5) Gull, Guys Hosp. Rep. V. (1859); ref. Schmidts Jahrb. Bd. 106. (1860). S. $291-294$.

6) $\mathrm{Hey}$, Inaug.-Dissert. Berlin 1898.

7) v. Hofmann, Wiener klin. Wochenschr. 1894. S. 823.

8) Humble, Lancet 1875. II. S 489 und 874.

9) Hutchinson, Transact. of the clin. soc. VIII. p. 127.1875; ref. Schmidts Jahrb. Bd. 173. S. 135.1877.

10) Kingston, Edinb. med, and surg. Journ. Vol.57. p. 69; ref. Schmidts Jahrb. 106. S. 294.

11) Köster, Göteborgs Cäkaresällks. förh. 1902. S.28; ref. Mendels Jahresber. 1902. VI. S. 558 .

12) Maclaren, Brit. med. Journ. 1897. I. S. 10.

13) Marchant, Bull. soc. anat. Paris. Bd. 51. S. 755.1876.

14) Mac Nalty, Lancet 1908. II. S. 1667.

15) Mecrkowski, ref. Mendels Jahresber. f. Med. V. 1901. S. 424.

16) Milian, Le liquide céphalo-rachidien. Paris 1904.

17) Möser, D. Arch. f. klin. Med. Bd. 35. S. 418. 1884.

18) y. zur Mühlen, Zeitschr. f. Ohrenheilkunde. Bd. 45. Heft 1. 
19) Nothnagel, Wien. klin. Wochenschr. 1902. S. 992.

20) Ohm, Deutsche med. Wochenschr. 1906. II. S. 1694.

21) Oppe, Münch. med. Abhandl. 1892. Heft 4 (a. a. Bollinger, Atlas d. path. Anat. I. S. 38. 1896).

22) Oppenheim, Lehrbuch der Nervenkrankheiten 1908.

23) Ord, Transact. path. soc. London. Bd. 32. 1881. S. 65.

24) Pfeufer, Ztschr. f. ration. Med. I. 1844. S. 293.

25) Ponfick, Virch. Arch. 1873. Bd. 58. S. 528.

26) Puzey, Lancet 1891. I. S. 368.

27) Reichmann, D. Ztschr. f. Nervenheilkde. Bd. 42. S. 1.

28) Rindfleisch, D. Arch. f. kl. Med. 1906 . Bd. 86. S. 183.

29) Roë, Transact. path. soc. London III. 1850/51. S. 46.

30) Rosenberg, Inang.-Diss. Greifswald 1904.

31) Russell, Brit. med. Journ. 1870. II. S. 87.

32) Saathoff, D. Arch. f. klin. Med. Bd. 84. S. 384.

33) Schalkhauser, Klin. Monatsbl. f. Augenheilkde. Bd. 16. Beil.-H. 2. 1878; ref. Schmidts Jahrb. Bd. 181. S. 177.1879.

34) Simmonds, Deutsche med. Wochenschr. 1901. S. 353.

35) Soucques, Iconogr. de la Salpêtr. 1908. Bd. 21. S. 108.

36) v. Steinmann, D. Arch. f. klin. Med. Bd. 13. 1874. S. 186.

37) Thyne, Lancet 1901. I. S. 397.

38) Tuffier s. Milian.

39) Uhthoff, Die Augenveränderungen bei den Erkrankungen des Grossund Kleinhirns. Leipzig 1911. 